\title{
Measurement of gas phase characteristics using a monofibre optical probe in a three-phase flow
}

\author{
P.C. Mena ${ }^{a, c}$, F.A. Rocha ${ }^{a}$, J.A. Teixeira ${ }^{b}$, P. Sechet ${ }^{c, *}$, A. Cartellier ${ }^{c}$ \\ a Department of Chemical Engineering, Faculty of Engineering, University of Porto, 4200-465 Porto, Portugal \\ ${ }^{\mathrm{b}}$ Center of Biological Engineering, IBQF, University of Minho, 4710-057 Braga, Portugal \\ ${ }^{c}$ LEGI, Laboratoire des Écoulements Géophysiques et Industriels, UMR 5519, UJF-CNRS-INPG, BP 53, 38041, Grenoble cedex 9, France
}

\section{A R T I C L E I N F O}

\section{Article history:}

Received 23 October 2007

Received in revised form 7 May 2008

Accepted 17 May 2008

Available online 24 May 2008

\section{Keywords:}

Bubble column

Solids

Optical probe

Gas holdup

Bubble rise velocity

\begin{abstract}
A B S T R A C T
The study of gas-liquid-solid systems structure requires reliable measurement tools. In this paper, preliminary results on the potential use of a monofibre optical probe to investigate such flow are presented. This probe, manufactured at LEGI, allows the simultaneous measurement of the gas phase residence time and gas phase velocity. This specificity makes this probe more interesting than classical single tip probes (which measure only the gas residence time) or double tip probes (which are more intrusive). Although extensively used in two-phase gas-liquid, this probe was never used in gas-liquid-solid systems. First, the probe signal response is studied for three-phase flow conditions in the presence of solids. Results show that for soft solids, the probe tips can be contaminated when the probe pierces the solid. The signal processing procedure was modified accordingly to take into account these events. Second, the probe results are validated by comparing global results (global void fraction, gas flowrate) deduced from profile measurements with measurements performed by independent means. Lastly, void fraction profiles and interfacial area are studied more in detail. Depending on the solid loading, these profiles exhibit different behaviours. These features are associated to characteristics of the flow such as the transition from an homogeneous regime to an heterogenous regime, and are consistent with global observation performed by independent means. This demonstrates the ability of the probe to connect local information to the global behaviour and structure of the flow.
\end{abstract}

(c) 2008 Elsevier Ltd. All rights reserved.

\section{Introduction}

Many research groups working with bubble columns (Jianping and Shonglin, 1998), slurry columns (Reese et al., 1996; Warsito et al., 1999; Li and Prakash, 2000; Xie et al., 2003), fluidized beds (De Lasa et al., 1984; Thompson and Worden, 1997), airlift reactors (Freitas and Teixeira, 2001), bubbly flows and flotation columns, are interested in the complex gas-liquid-solid systems. Their wide application in various industrial processes such as chemical, petrochemical, biochemical and environmental is a strong evidence of their increasing importance. Despite all the research efforts, the knowledge about the effects of solids on gas-liquid system and the respective physical mechanisms are not yet clarified. A clear understanding of the hydrodynamics of the gas-liquid-solid systems is needed to improve

\footnotetext{
* Corresponding author. Tel.: +33476825041; fax: +33475825271.

E-mail address: philippe.sechet@hmg.inpg.fr (P. Sechet).
}

the design and operation of the processes involving these systems. It is particulary true in the field of bioengineering where three-phase flow reactors, such as three-phase airlift reactors with immobilized biomass, are encountered.

The presence of the solid phase can influence the gas-liquid mixture in different ways such as bubble rise and formation, radial (Warsito and Fan, 2001) and axial profiles, mixing and dispersion, gas holdup and flow regimes (Zhang et al., 1997; Mena et al., 2005b), mass transfer (Sada et al., 1985; Joly-Vuillemin et al., 1996; Mena et al., 2005a). Thus, a reliable measurement technique is needed to have a better insight of the local flow structure and a better understanding of the various interactions between phases. Among all the available instrumentation, phase detection probes are frequently used in the investigation of two-phase flows (Cartellier, 1998) and more recently also in three-phase flows (Yang et al., 2001; Schweitzer et al., 2001; Boyer et al., 2002; Wang et al., 2003). Most sensors are based on conductivity, capacitance, optical or temperature measurements (Boyer et al., 2002). Recently, a new sensors based on complex permittivity measurement has also been developed and successfully 
employed in an air/water/gasoline flow (Silva et al., 2007). Among all these technologies, optical probes are frequently used. Nowadays optical probes are able to measure not only phase concentrations but also bubble velocities, size distributions (Liu et al., 1998), mean interfacial area, mean Sauter diameter (Leung et al., 1995; Kiambi et al., 2001). They are able also to identify flow regimes (Kozma, 1995). This information is of crucial importance to the description and modelling of multiphase flows. These characteristics, combined with their relatively simple operating principle and use make the intrusive phase detection probes very attractive for industry and research. As these probes were primarily developed in the frame of gas-liquid or liquid-liquid flows (Fordham et al., 1999), their application to flow with solids must be performed with caution. In the present work, we investigate the ability of a new generation of optical probes, manufactured in LEGI, to perform measurements in such complex flow. The technique's specificity consists in the possibility to measure at the same time the local void fraction and the bubble velocity using a unique single tip probe (Cartellier, 1992). These probes are very promising: the spatial resolution is increased allowing for a more refined description of the flow compared to classical optical probes. However, as shown in Cartellier and Barrau (1998b) the measured quantities, in particular the bubble velocity, depends on the complex interaction between the inclusions and the probe tips. So, apart from the question of the probe fragility, the main concern with the use of optical probes in three-phase flows is the possible modification of these interactions and their effects on the measurement accuracy.

The paper is organized as follows. In the first part, we summarize briefly the basic operation of optical probes and the specificity of LEGI's probes. The various sources of error arising when the probe is used are also summarized. In the second part, solid/probe interactions are studied, coupling the probe measurement with high-speed camera recording. The aim is to analyse the probe response and deal with possible changes on the signal due to solid/probe interactions. In the third part, the ability of the probe to perform measurement in three-phase conditions is analysed on a flow. The acquired data are compared to global quantities measured by other means and the ability of the probes to capture key features of the flow is shown.

\section{Multiphase flow measurement using optical probes}

\subsection{Generality}

Optical probes are used to locally detect the presence of the gas phase in a multiphase system. A monochromatic light is transmitted through an optical fibre to the tip. When the tip is dipped into a gas phase, the light is mainly reflected, travels back to the detector through a $\mathrm{Y}$ junction and is converted into an electrical signal (high level signal). When the tip is immersed in a liquid environment, the light is scattered and almost no light is reflected back to the emitter/receiver device leading to a weak electric signal (low level signal) (Boyer and Cartellier, 1999). The time dependant signal consists in a succession of crenel shaped transitions. The signal processing, when properly done, leads to the definition of a phase indicator function $X: X=1$ if the probe is in the gas phase and $X=0$ if the probe is in the liquid phase (see Fig. 1).

The duration of each individual crenel, $T_{G}$, is representative of the residence time of a given inclusion at a given point. The local void fraction is then measured taking the ratio between the total duration for which the probe is in the gas over the total acquisition time $t_{\mathrm{acq}}$

$\varepsilon g=\frac{\sum T_{G}}{t_{\mathrm{acq}}}$

Let us notice that the signal is creneled shaped and reach its maximum for "well" pierced bubbles. When the piercing is done too close
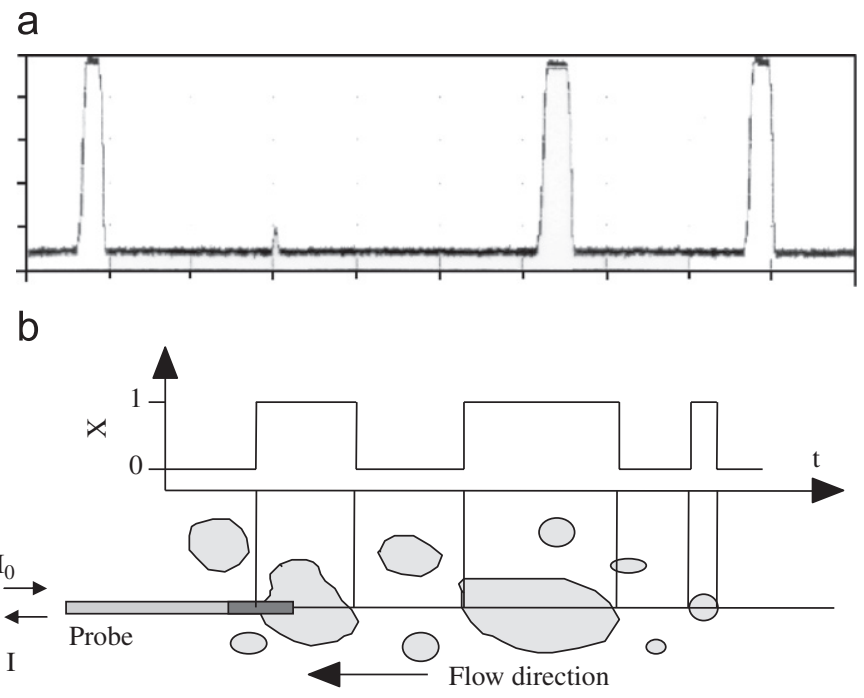

Fig. 1. (a) Typical recorded signal and (b) indicator function. $I_{0}$ : intensity of transmitted light, I: intensity of reflected light.

from the bubble side, because of film deformation and drainage properties, sometimes, individual events may have an amplitude below the maximum gas level. In the sequel, for normal operation conditions, the "mean gas level" will be defined as the value of the maximum signal level averaged over sufficient number of events (in order to get rid of fluctuations due to noise from the electronic for instance).

For first generations single-tip probes, only $T_{G}$ is accessible. In order to access more refined quantities, the bubble velocity is needed. This velocity can be measured using, for instance, a double-tip probe. Knowing the axial distance between the two tips, for a given inclusion, the time lag between crenels on each signal allows the velocity calculation.

\subsection{Description of LEGI's optical probes}

As an intrusive technique, double-tip probes suffer from some limitations: in particular, the measurement volume (spatial resolution) is not that small and the inclusion interaction with multiple tips can make the signal processing difficult. The single tip probes manufactured in LEGI allows to overcome such limitations. First, the probe does not affect much the flow structure due to its small size (fibre diameter like $150 \mu \mathrm{m}$ ): this increased resolution allows a more refined description at small scale (microstructuration). Second, it is possible to link the rising (or transition) time $T_{m}$ between the low level signal and the high level signal to the inclusion velocity.

The relationship between the rising time $T_{m}$ and the bubble velocity $V_{b}$ was extensively studied in Cartellier and Barrau (1998a) and Cartellier and Barrau (1998b). To summarize their work, the analysis of liquid to gas transition across well controlled interface showed that their transition $T_{m}$ is inversely proportional to the interface velocity $V$ when the probe is normal to the interface. The transient can be then interpreted as the translation of an idealized plane interface along a characteristic length $L$, called latency length: $L=V \cdot T_{m}=$ constant for a given probe. The latency length is a characteristic of the probe. If $L$ is known, then the rising time knowledge allows the velocity computation.

In order to make accurate velocity measurement using the above principle, probe optimization is required. It was shown (Cartellier and Barrau, 1998a, 1998b) that specific geometry of the sensitive part of the tip gave more accurate results. This led to the design of two type 
of probes: conical probes (1C probes) and conical-cylinder-conical probes ( $3 \mathrm{C}$ probes). The probe used in this paper is a $1 \mathrm{C}$ probe (see Fig. 2).

Although the latency length is a characteristic of a given probe, it is not easily predictable when the probe is manufactured. That is why each probe needs a calibration.

Furthermore, when the probe is calibrated (or the raw signal is processed), rise times are defined by setting a lower and upper thresholds on the signal amplitude so that the very beginning and very end of the transitions are ignored. This procedure allows to get ride from some parasitic phenomena on the signal (for instance, proximity detection Cartellier, 1992; Cartellier and Barrau, 1998a). These thresholds are defined as a percentage of the amplitude gap between the constant liquid and gas levels (see Fig. 3). The effect of this threshold on the latency length is also presented in Cartellier and Barrau (1998a). For a given probe, calibration must then be given along the threshold used to perform the calibration curve. For most applications, threshold value of $10 \%$ and $90 \%$ are reliable to calculate the rising time. These are the values which have been used for the probe used in this paper. Fig. 3 present a sketch of a typical signal for a $1 \mathrm{C}$ probe. All the parameters defined above are included in the

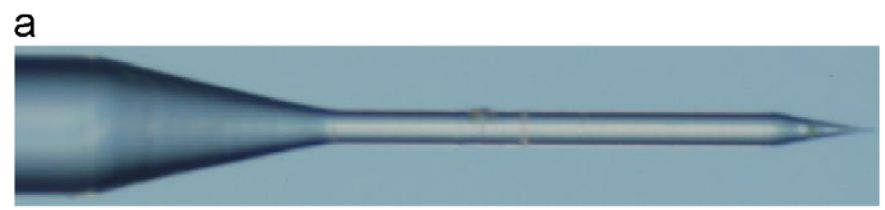

b

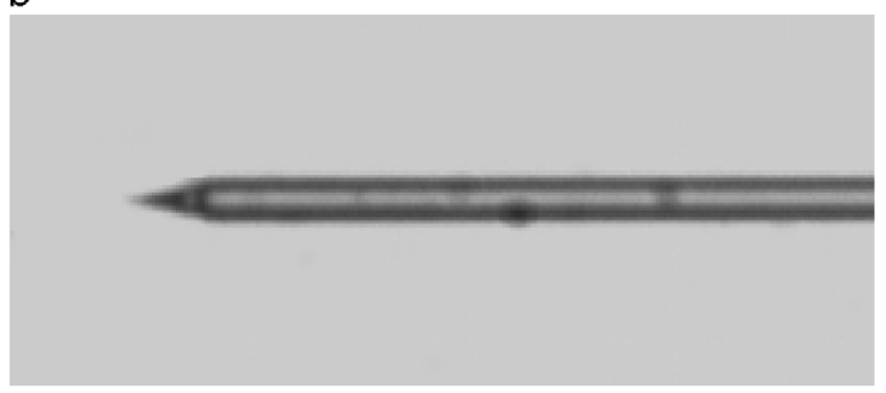

Fig. 2. Probes geometries (a) $3 C$ and (b) $1 C$. figure. The probe calibration curve for the probe used in this paper is presented Fig. 4.

\subsection{Measurements error using optical probes in two-phase flow configuration}

In order to have a frame of reference when results will be analysed for three phase flow configurations, we summarize various error sources arising when optical probes are used in two-phase dispersed gas-liquid flows. Error on the void fraction arise from the measurement of the residence time $T_{G}$. Different processes have been identified to explain these errors (Julia et al., 2005; Cartellier, 1992; Cartellier and Barrau, 1998a): (i) the "blinding" effect, when small chords are not detected due to imperfect tip dewetting, (ii) the "drifting" effect when the bubble trajectory is altered leading to a smaller chord detection or no detection at all and (iii) the "crawling" effect due to

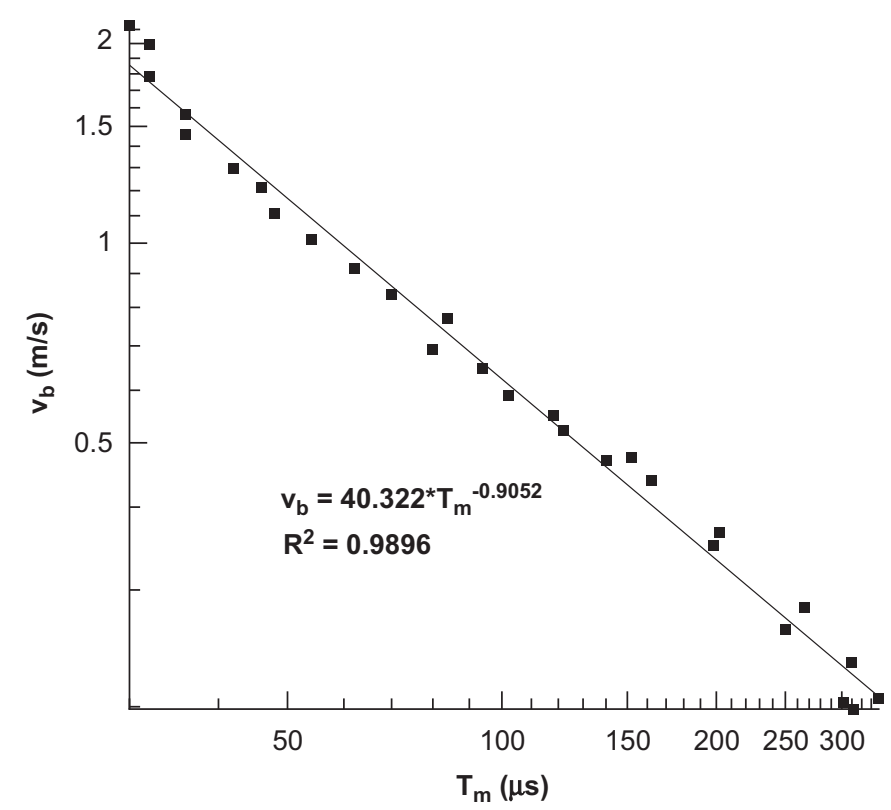

Fig. 4. Calibration curve of the monofibre optical probe used in this paper.

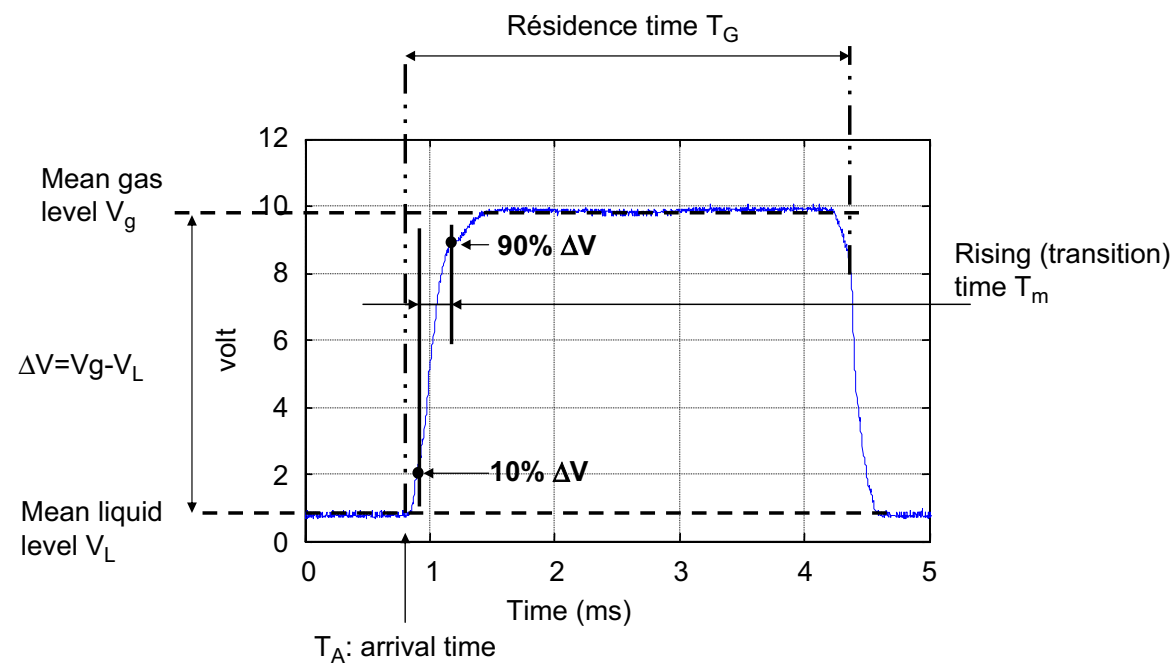

Fig. 3. Signal example of a bubble signature and definitions. $T_{G}$-residence time, $T_{m}$-rising time, $T_{A}-$ bubble arrival time, $V_{g}-$ mean gas level, $V_{L}-$ mean liquid level. 


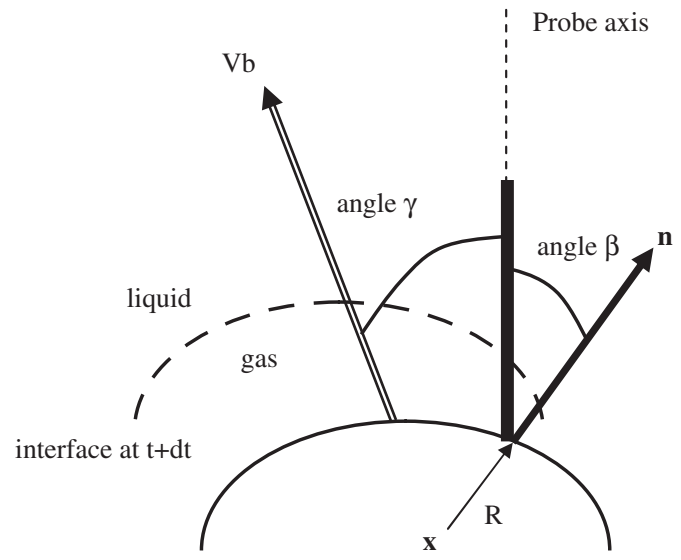

interface at $\mathrm{t}$

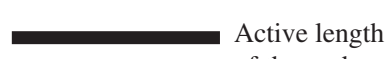
of the probe

Fig. 5. Definitions of the angles $\beta$ and $\gamma$. From Cartellier (1992).

bubble deformation and deceleration on the probe tip. This various effects have been quantified on controlled idealized cases (Julia et al., 2005; Cartellier and Barrau, 1998b; Carrica et al., 1995). Blinding and drifting effect leads to an underestimation of the residence time. At the opposite, the crawling effect leads to an overestimation of the residence time. As bubble piercing is a random process, it is difficult to predict and quantify the contribution of each process on the error for complex 3D flows. However, measurements performed by Cartellier and Barrau (1998b) on a "laminar" bubbly flow in column with a $1 \mathrm{C}$ probes led to relative errors on void fraction ranging from $1 \%$ to $15 \%$, the average error beingaround $5 \%$ on the global void fraction. The error increase at low void fraction and low liquid velocity (drifting effect and blinding effect).

The error arising from the relationship between $T_{m}$ and $V_{b}$ was studied in Cartellier (1992) for stretched probes and Cartellier and Barrau (1998a), for conical and 3C probes. The rising time is very sensitive to the angle $\beta$ (between the probe and the normal to interface), angle $\gamma$ (between the probe and the bubble velocity vector) and possibly to the interface radius of curvature $R$ (Fig. 5).

In Cartellier (1992), a specific study was done for stretched probes with $\gamma=0^{\circ}$ and variable $R$. Results showed that the rising time was insensitive to $R$ (over the range investigated, i.e. $2-7 \mathrm{~mm}$ ). Specific studies on the influence of $\beta$ were also performed (Cartellier, 1992; Cartellier and Barrau, 1998b). Results showed that $T_{m}$ and the latency length were weakly sensitive to $\beta$ when $\beta<10^{\circ}$ but the rising time increases quickly above this angle (for the same operating conditions) until $\beta=30^{\circ}$. Above this angle, the interface is distorted, the rising time begin to decrease and cannot be taken to be a characteristic of the interface velocity. It is thus unrealistic to find a correlation of practical use under the form $T_{m}$ (or $\left.L\right)=f(V, \beta, \gamma, R)$ as it is impossible to know the angle of impact in realistic flow. However, as the correlation $V=f\left(T_{m}\right)$ is weakly sensitive to $R$ and $\beta$ for small $\beta$ the velocity can still be measured for samples where the impact is quasi-perpendicular to the probe. This is performed by discriminating bubbles whose signature on the signal correspond to a quasiperpendicular piercing. For events whose the rising time $T_{m}$ is not recorded, the velocity is interpolated. The signal processing principle can be found in Cartellier (1992) with some qualification tests. This algorithm was improved in Barrau et al. (1999) to allow real time signal processing. This is this later algorithm which is implemented in our software.

Another source of error could be the cleanliness of the probe. This question was addressed (although not extensively) in Cartellier and
Barrau (1998a). Results shows that the rise time between the $10 \%$ and $90 \%$ level remains nearly the same (study performed for two impact angles, namely $0^{\circ}$ and $30^{\circ}$ ).

Given these error sources, the estimation of uncertainties on velocities was made for a stretched probe by Cartellier (1992) and for a 3C probes by Barrau et al. (1999). In Cartellier (1992) extreme errors on velocities for bubbles were around $20 \%$. On the same experimental set-up, Barrau et al. (1999) found that the errors on the flow rate were $\pm 10 \%$ except for very low gas fraction. In that later case the over estimation on the flowrate is around $16 \%$ for liquid superficial velocity equal to $0.2 \mathrm{~m} / \mathrm{s}$ and $35 \%$ for stagnant conditions.

Finally, keeping in mind the restrictions presented above, bubble concentration, velocity and bubble size are available at the same time with a unique single probe. These probes have been successively used in various configurations to study bubbly flow (Rivière et al., 1999) or atomization (Hong et al., 2004). Not only quantities such as the void fraction profiles can be measured but also quantities related to the local structure of the flow (Cartellier and Rivière, 2001). For instance, in the case of bubbly flow, the spatial organization of the flow at small scales (including wall peaking effect, clustering formation, induce agitation) could be quantified (Cartellier and Rivière, 2001; Rivière et al., 1999). So far, these probes were only used in two-phase configurations (bubbly flow, slug flow, spray flow). As mentioned in the Introduction, their extension to three-phase configurations has a considerable interest in the scope of studying the alteration of the flow structure and mixing due to the solid phase. However, the ability of the probes to give accurate results in such configurations has still to be demonstrated. Indeed, the interaction between the probe and the solid phase can lead to inaccurate measured values and false bubble detection. Furthermore, the signal processing may need improvement as the interaction of inclusions with the probe are more complex. Lastly, the probe is fragile and it must be checked if it can support frequent contacts withsolid material. The objective of the work presented in the next section is to perform these tests and give a first answer on the ability of the probes to function and give reliable results in this kind of configuration.

\section{Validity of the optical probe response for three-phase systems}

\subsection{Experimental conditions}

The measurements were carried out in a cylindrical plexiglas bubble column of diameter $D_{c}=0.072 \mathrm{~m}$ and height $H_{c}=0.5 \mathrm{~m}$. The column was equipped with a perforated plate with $0.6 \mathrm{~mm}$ orifices and relative free area ratio $(\varphi) 1 \%$. Compressed air from laboratory lines was the gas phase and tap water was the liquid phase. The solids used were calcium alginate beads. Calcium alginate beads are roughly spherical particles. In these experiments, their equivalent diameter was $d_{p}=2.1 \mathrm{~mm}$ and density $\rho_{p}=1023 \mathrm{~kg} / \mathrm{m}^{3}$. The choice of this solid phase corresponds to our interest in three-phase airlift reactors with immobilized biomass. The solids are well-defined completely wettable objects with reasonable rigidity that do not form agglomerates and are big enough not to affect the surface properties of the gas-liquid interface.

The on-line acquisition data was done using the software So2_4 developed at LEGI. For each experiment this software recorded the rising time $\left(T_{m}\right)$, the residence time $\left(T_{G}\right)$ and the arriving time $\left(T_{A}\right)$. For some experiments vizualization facilities such as an high-speed camera synchronized with the signal acquisition were used. This later configuration is presented on Fig. 6 .

\subsection{Probe response analysis in gas-solid-liquid flow}

The software So2_4 used to process in real time the optical probe signal was modified in order to record not only the arrival time, 


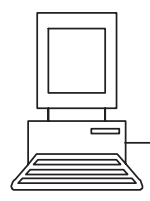

7

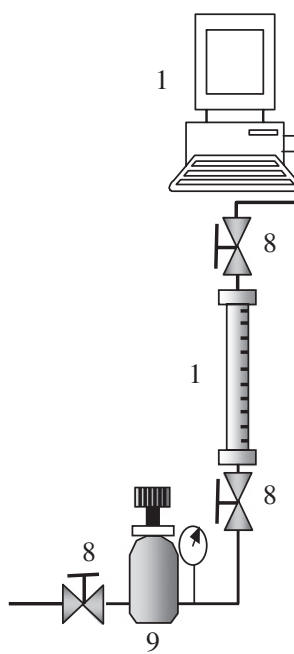

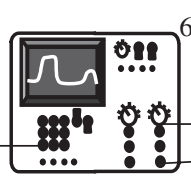

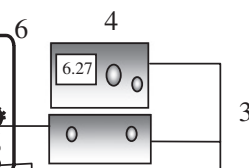

\section{5}

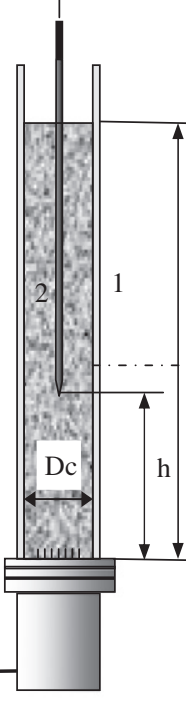

H

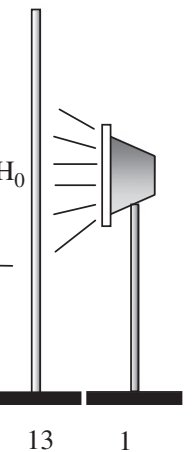

Fig. 6. Experimental set-up for optical probe measurements: 1-bubble column, 2-optical probe, 3-Y junction, 4-light emitter, 5-light receiver and converter, 6-oscilloscope, 7-PC1, 8-on/off valve, 9-pressure reducer, 10-rotameter, 11-high-speed camera, 12-PC2, 13-diffuser glass and 14-halogen lamp. the residence time and the rising time for each event, but also the amplitude of the signal of each event. Preliminary measurements showed that the probe was able to work properly up to high solid content but with the calcium alginate beads, the signal presented some abnormality: in this case, the signal showed peaks with higher amplitudes, besides the peaks of normal amplitudes.

This phenomena is clearly seen on Fig. 7. This figure shows the normalized amplitude distribution of events for different alginate beads loading ((a) $0 \%$, (b) $5 \%$, (c) $15 \%$ and (d) $25 \%$ ). The gas flowrate is $6.6 \mathrm{l} / \mathrm{min}\left(u_{G}=2.7 \mathrm{~cm} / \mathrm{s}\right)$. By normalized amplitude we mean the amplitude of the signal for a given event divided by the mean gas signal level for a normal bubble detection. Indeed, depending on the tuning of the electronic and other factors such as the probe cleanliness, the mean gas level for the gas phase can be different from one run to the other. This procedure allows to make easier comparisons.

It is clear from that figure that as soon as there are solids, the distributions become bimodal, one mode corresponding to normal detection and one mode corresponding to maximum abnormal peak amplitude. Furthermore, there are events whose amplitude are comprised between these two reference levels, and their number increase with the solid loading. Thus the questions are: (i) to which kind of events correspond normalized amplitude above 1, (ii) does the distribution behaviour correspond to the probe response to every kind of solids or is it a particular behaviour of alginate beads and (iii) how to discriminate solid-tip interaction events from normal bubble events. Even if the paper is centred on the alginate beads case, some tests were performed with other kinds of solids to check if this abnormality was particular to alginate beads. Solids used were glass beads a

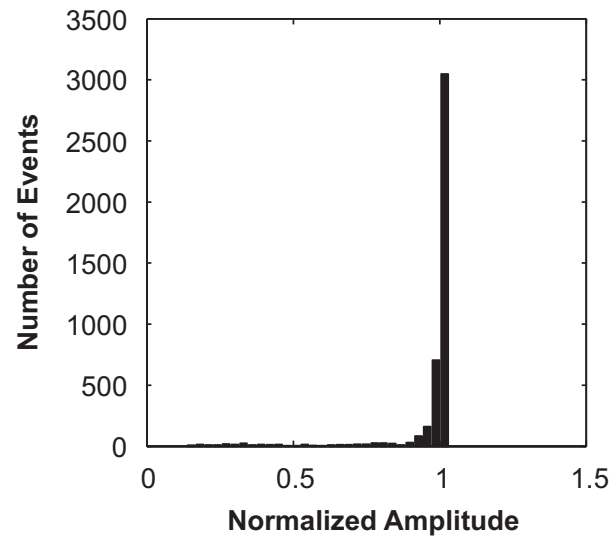

C

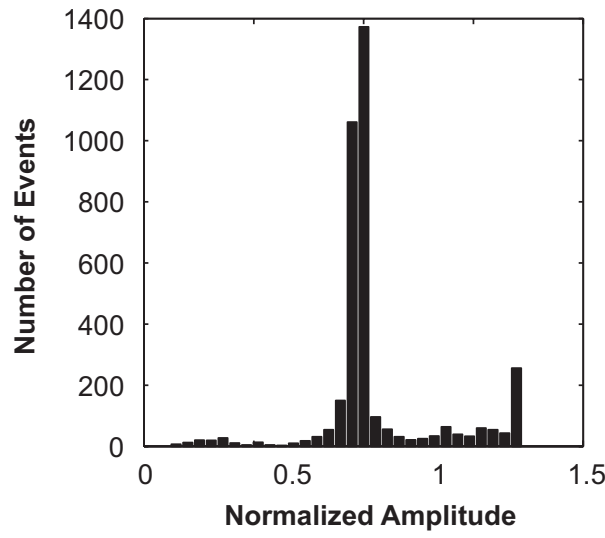

b

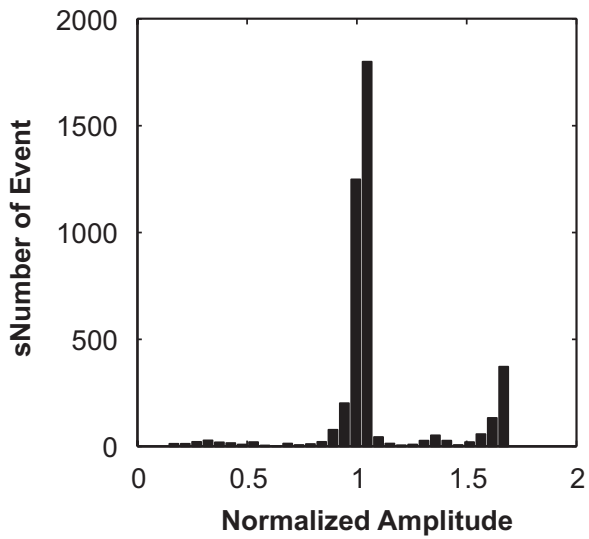

d

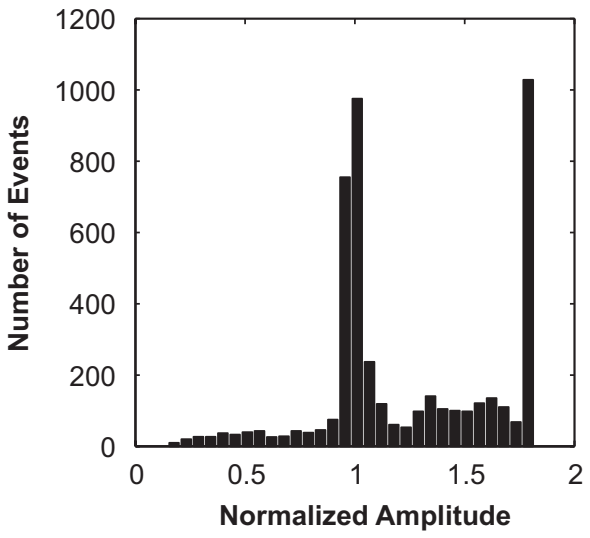

Fig. 7. Distribution of the signal normalized amplitude of detect events. Alginate beads, $u_{G}=2.7 \mathrm{~cm} / \mathrm{s}$ : (a) $0 \%$ solids, (b) $5 \%$, (c) $15 \%$ and (d) $25 \%$. 
a

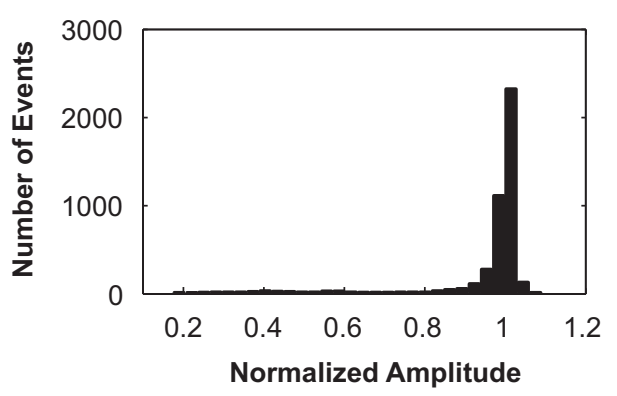

C

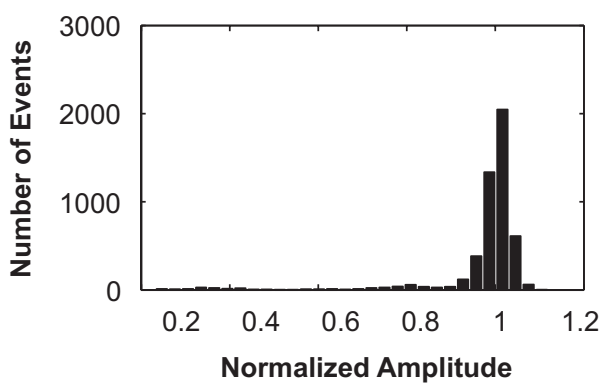

e

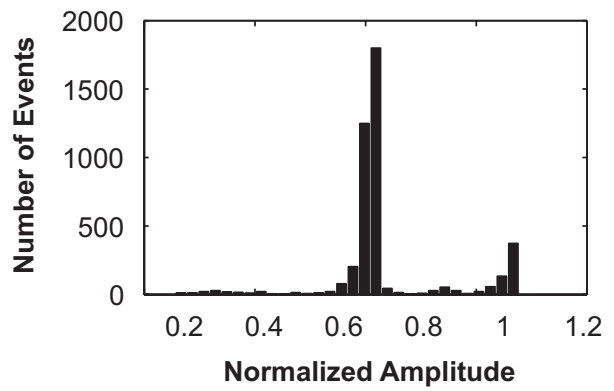

b

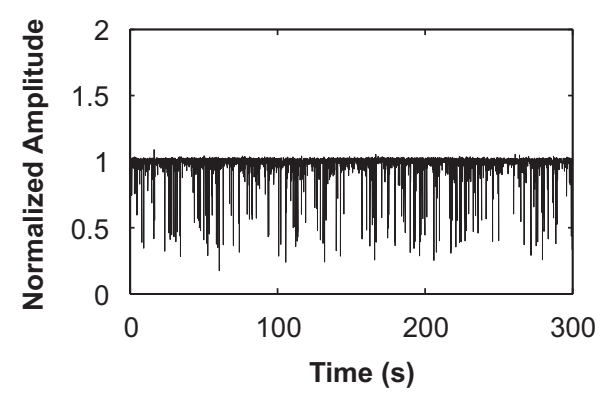

d

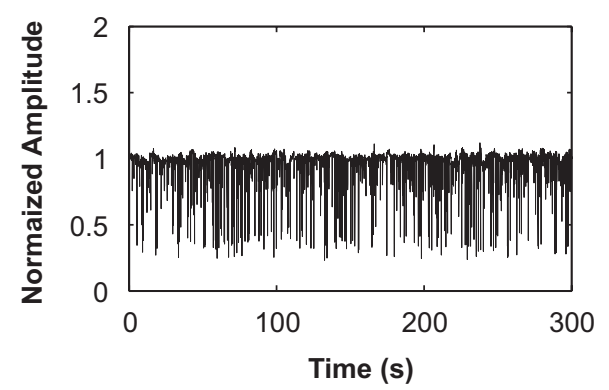

$\mathrm{f}$

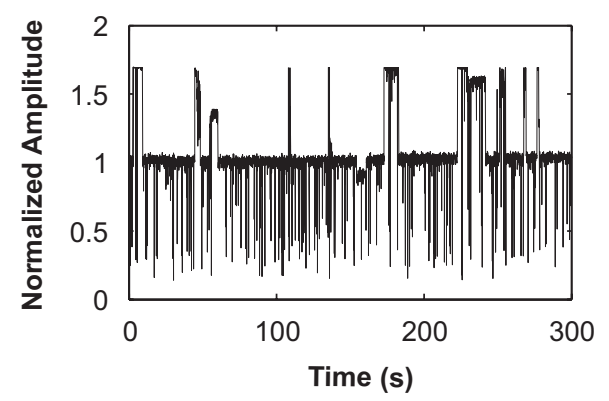

Fig. 8. Time series of signal amplitude and signal amplitude distribution (a) and (b) glass; (c) and (d) polystyrene; (e) and (f) alginate.

$\left(d_{p}=80 \mu \mathrm{m}, \rho=1.1 \mathrm{~g} / \mathrm{cm}^{3}\right)$ and polystyrene beads $\left(d_{p}=1100 \mu \mathrm{m}\right.$, $\left.\rho=1.05 \mathrm{~g} / \mathrm{cm}^{3}\right)$.

Figs. 8(b), (d), (f) show the time chronicle of the normalized amplitude of each recorded event versus the arrival time of the events. Figs. 8(a), (c), (e) show the corresponding distribution of the normalized amplitudes.

The solid loading is $5 \%$ and superficial gas velocity still $2.7 \mathrm{~cm} / \mathrm{s}$ as we did not make extensive test with glass and polystyrene for all the solid loading ranges and superficial gas velocities studied in the work with alginate beads. However, the figure shows typical behaviour for each kind of solids.

First of all, the high amplitude peaks and bimodal distribution are present only for alginate beads. Second, even for low solid loading, for the two other kind of solids, there exists some "noise" (by noise, we understand mean signal gas level fluctuations resulting in events whose normalized amplitude is slightly greater than one).

To analyse the specific behaviour of the alginate beads and clarify whether the high intensity probe signals, observed in the three-phase system with alginate beads, represent bubbles or solid particles in contact with the optical probe tip, a high-speed camera, recording at $1000 \mathrm{fps}$, was synchronized with the optical probe signal acquisition in order to ascribe a certain probe signal to the respective event. A pretrigger enables image recording to start before the first high amplitude signal is detected. Several tests were performed, varying the solid loading, superficial gas velocity and the trigger level.
The results presented here correspond to the experiments performed at $u_{G}=1.6 \mathrm{~cm} / \mathrm{s}, 5 \mathrm{vol} \%$ of solids and trigger level of $2 \mathrm{~V}$. In Fig. 9 a typical probe signal is presented and the respective highspeed camera image sequences are shown in Fig. 10. When a calcium alginate particle is pierced by the optical probe, the probe tip is contaminated with small solids which attach to the probe. Due to nature of the solids (porous gel) when the calcium alginate beads hit the probe small sticky and soft parts are deposited on the probe. This results in a high amplitude signal which is immediately detected by the probe (see signal A in Fig. 9 and sequence A1-A3 in Fig. 10). When the tip is "contaminated" by small (white) particles the light reflection increases, leading to high amplitude signal. Fig. 11 is an example among many other. In this case the recovery process was $4.618 \mathrm{~s}$ long, while in other cases this time was different (for instance 1 or $2 \mathrm{~s}$ approximately). Due to the probe tip contamination, the next bubbles piercing also exhibit high amplitude $(\simeq 3.4 \mathrm{~V})$, comparing to the normal amplitude $(\simeq 1.3 \mathrm{~V})$ for bubble detection (see signals $B$ and $C$ in Fig. 9 and sequences B1-B3 and C1-C3 in Fig. 10). The long time probe signal acquisition shown in Fig. 11 seems to demonstrate that the high amplitude signal resulting from a solid piercing, which contaminates the probe, is kept for the next bubbles piercing and then the signal stabilizes (for $t=4.618 \mathrm{~s}$ on the example presented Fig. 11) at the typical bubble detection amplitude values, meaning that the small contaminants were detached and the probe is clean. 
The number of run with the high-speed camera were limited as the technique is not applicable for a high content of solids and/or gas. That is why data were analysed from two other different points of view in order to complete the analysis performed from the visualizations.

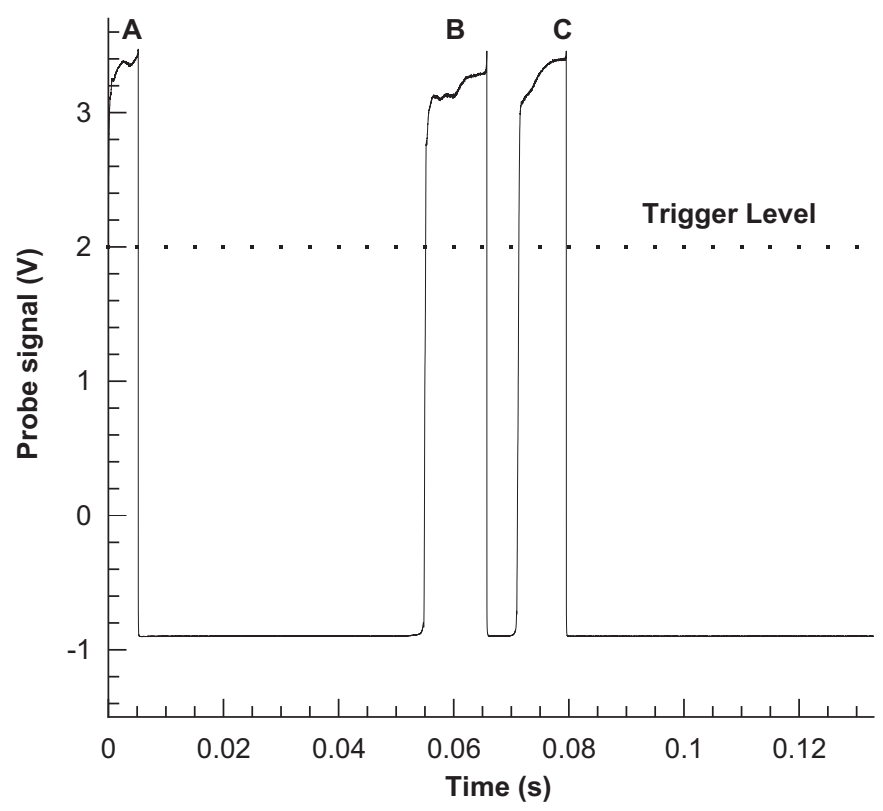

Fig. 9. High amplitude signal observed for bubbles after probe tip hitting by solid: A-solid piercing (seq. A1-A3 in Fig. 10). B and C-bubble piercing (seq. B1-B3 and C1-C3 in Fig. 10).
A first point of view, which seems to contradict at the first sight the probe contamination assumption presented above, consists in considering that all peaks above a given threshold are solid/tip interactions. Fig. 12(a) shows for $5 \%$ solid loading, the evolution of the measured void fraction with the threshold used to discriminate events (for a given threshold, only events whose amplitude is

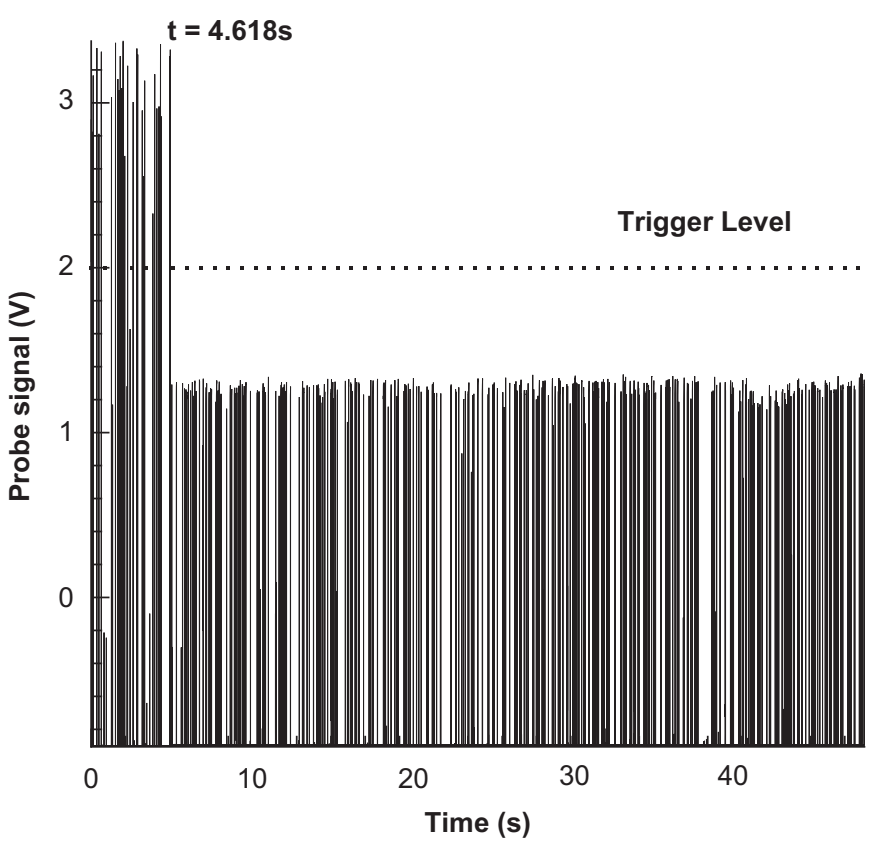

Fig. 11. Probe signal during particle and bubble piercing (long time acquisition)
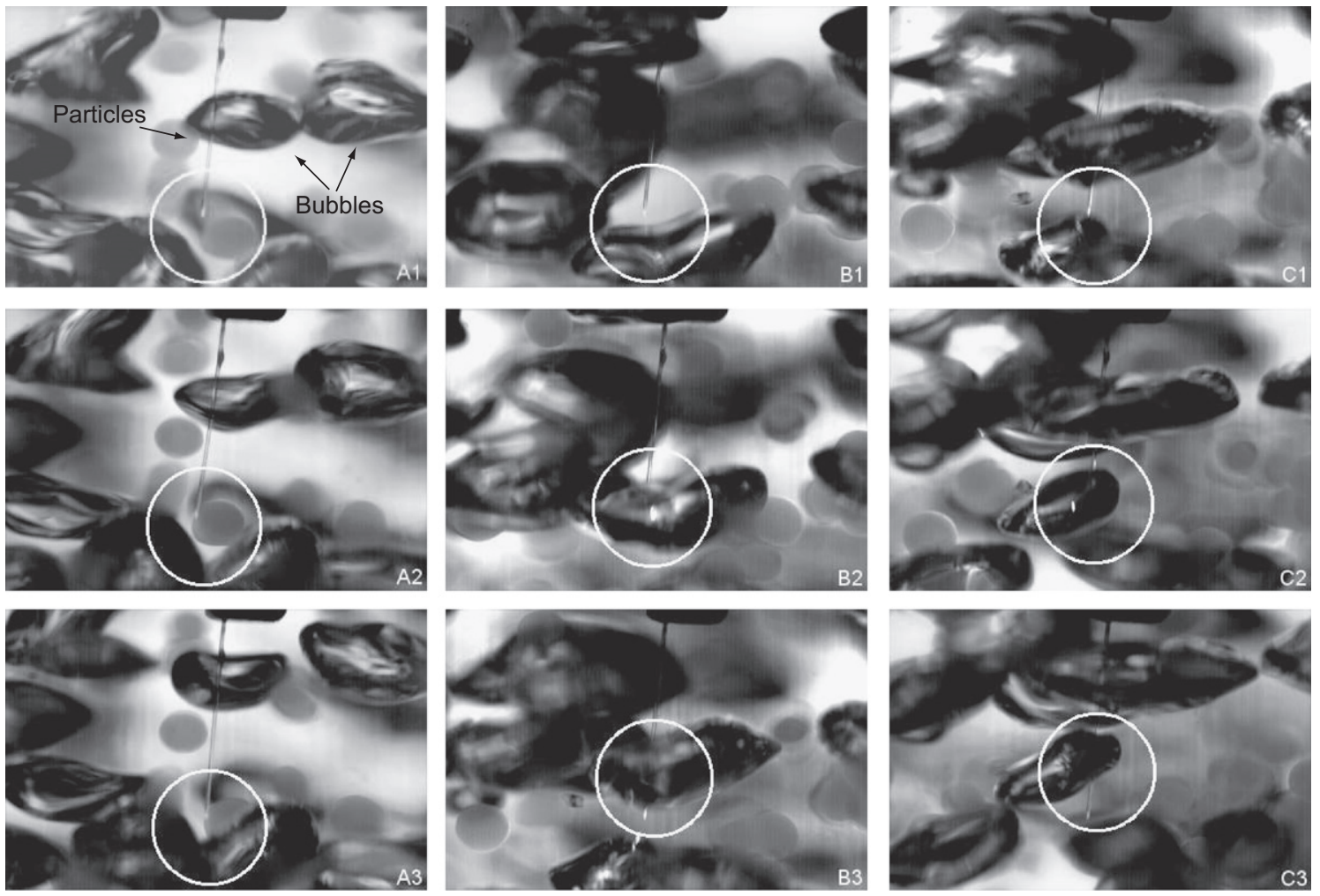

Fig. 10. High-speed camera image sequences of particle and bubble piercing: A1-A3 particle piercing; B1-B3 bubble piercing; C1-C3 bubble piercing. 
a
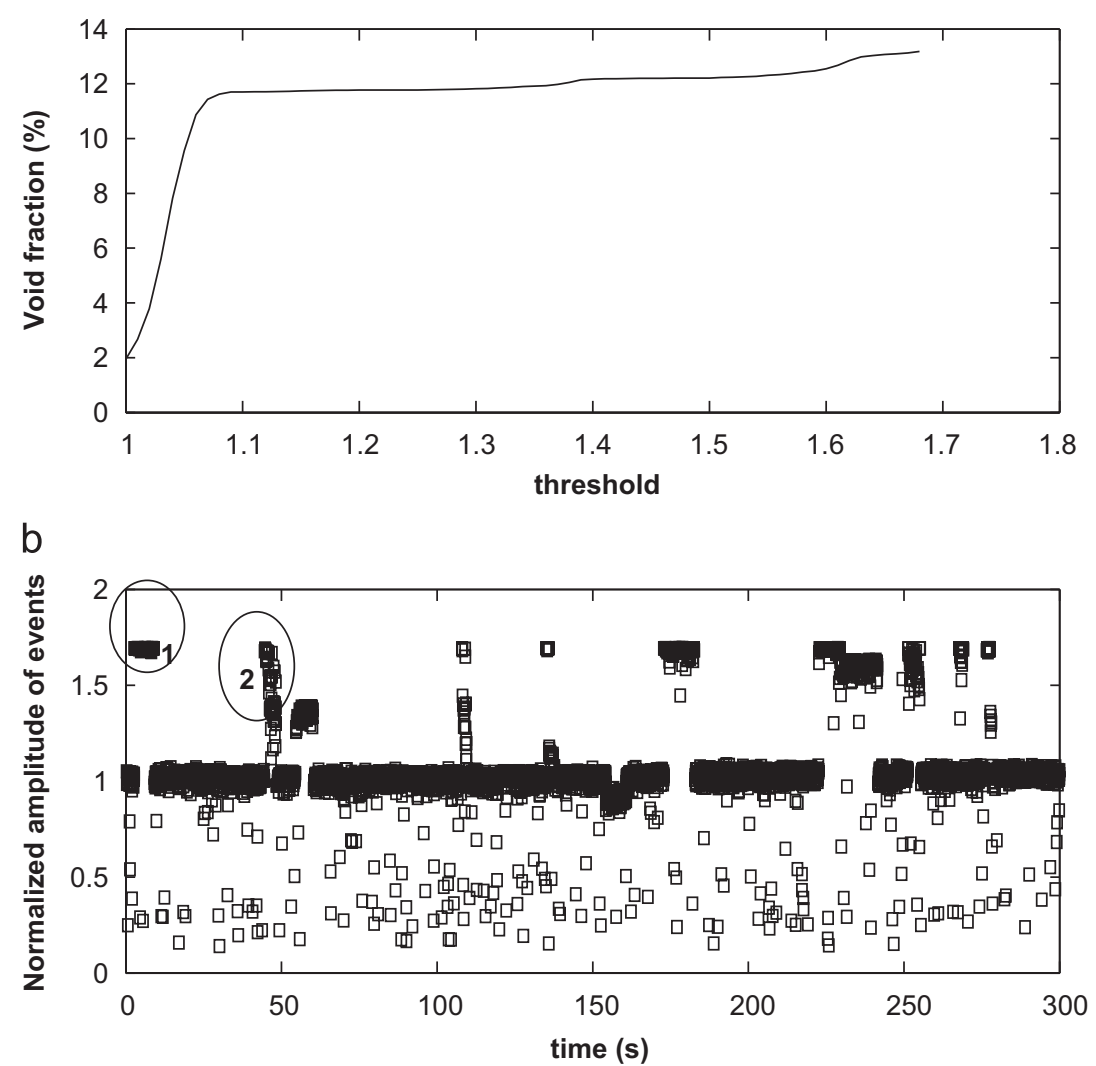

Fig. 12. Alginate beads, $5 \%$ loading, $U_{g}=2.7 \mathrm{~cm} / \mathrm{s}$. (a) Local void fraction evolution with the threshold. (b) Corresponding time series of normalized amplitudes.

below the threshold are kept to calculate the void fraction). Fig. 12(a) is presented along the corresponding normalized amplitude time series (Fig.12(b)). The small loading was chosen to ensure that the flow was not too disturbed, with no recirculating cells due to large scale instabilities and a flat void fraction profile. It allows a rough comparison between the local void fraction measured by the probe and the global gas holdup, measured by bed expansion for instance, without having the knowledge of the complete local void fraction profile. In the case of Fig. 12, the gas superficial velocity was $u_{G}=$ $2.7 \mathrm{~cm} / \mathrm{s}$ and the gas holdup measured by bed expansion was equal to $14 \%$.

It is interesting to see that for low threshold (for instance < 1.2), the measured void fraction increase drastically with the threshold level. From threshold ranging from 1.2 to 1.8 (the maximum amplitude of detected events), the void fraction evolution is far slower (between $12 \%$ and $14 \%$ ). This means that even if the number of high amplitude event seems small in Fig. 12b, their contribution to the void fraction is not negligible (in this example, on 4284 detected events, 703 have an amplitude 1.2 times greater than the normal gas level). Indeed, for this configuration, as it will be seen later, the void fraction profile is nearly flat and the local void fraction is a good estimate of the gas holdup. It is hardly believable that the error on the gas holdup measurement performed by bed expansion leads to an overestimation equal to 5 or 7 times the real void fraction. This leads to think that above a ratio of 1.1-1.2, events include solid/tip interactions but are in majority bubble events. This confirms the analysis presented from the visualization at least for low solid loading.

Fig. 12b shows also an interesting feature of the time series behaviour. The events in circle 1 corresponds to the same kind of events presented in Fig. 9, with a sudden recovery of the signal amplitude to the normal gas level. However, events in circle 2 present a progressive recovery. This comfort the tip contamination assumption. The probe latency length is small (120-150 $\mu \mathrm{m}$ usually) and the probe sensitive part can be easily entirely covered by material detached from the alginate beads. Depending on the size and number of the contaminating elements, the recovery can be sudden or progressive depending on how fast the probe is cleaned and how much material is removed at a given time. This observation is consistent with the distributions shown in Fig. 7: the number of event whose normalized amplitude is in the range $(1,1.8)$ increase with the solid loading as the contamination probability increase but the majority of this peaks correspond to a progressive recovery of the probe cleanliness (i.e bubbles events of abnormal amplitude).

To complete the argumentation presented above, the effect of threshold was analysed using a procedure based on the results obtained from the high-speed camera visualization: only the first peak in a group of several high amplitude peaks is considered as solid/tip interaction and is discarded. A group of such peaks is considered to be detected when the normalized amplitude of an event is above a fixed threshold $\mathrm{TH}$. The end of a group is detected when the normalized amplitude goes below 1.1. The effect of the threshold level $\mathrm{TH}$ on the void fraction is then examined. Results are presented in Fig. 13 for different solid loading: (a) 5\%, (b) $15 \%$ and (c) $25 \%$. The gas superficial velocity is $u_{G}=2.7 \mathrm{~cm} / \mathrm{s}$. There is a clear distinction on the void fraction evolution for threshold values below a given critical value $\mathrm{TH}=1.1$ or above this critical value. The fast evolution of the void fraction with the threshold below $\mathrm{TH}=1.1$ means that the number of discarded events decrease quickly with TH. This correspond to isolated events. From the results presented in Fig. 12 these events are bubbles as the void fraction is too low compared to the gas holdup measured by independent means. Above $\mathrm{TH}=1.1$, the void fraction evolution is far slower which corresponds to few events 
a
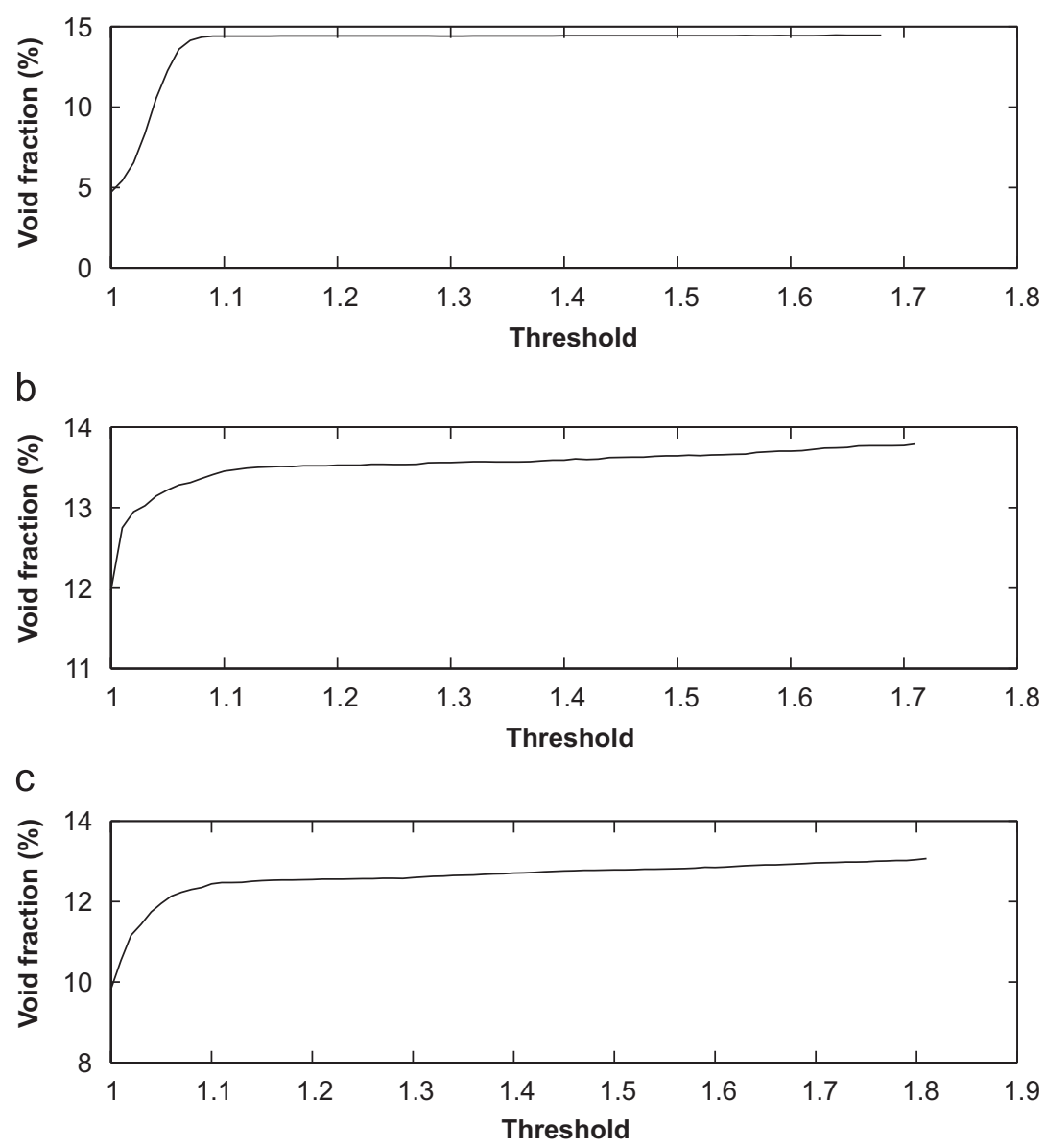

Fig. 13. Void fraction evolution with threshold TH. Alginate beads: (a) 5\% loading, (b) $15 \%$ loading and (c) $25 \%$ loading.

which are discarded. This is consistent with the concept of grouped amplitude peaks, where only the first event is discarded.

The maximum deviation on the void fraction computed for threshold value $\mathrm{TH}=1.1$ and for a threshold $\mathrm{TH}$ corresponding to the maximum amplitude in a run is $0.3 \%$ for $15 \%$ loading and $0.4 \%$ for $25 \%$ loading, which corresponds, respectively, to a relative error of $2 \%$ and $3 \%$. For $5 \%$ loading, the error is hardly noticeable. These observations are consistent with the amplitude distributions shown in Fig. 7 for glass and polystyrene which show that compared to the case of $0 \%$ solid loading, there are events whose level is slightly above the mean gas level. From results obtained with alginate beads these events are considered to be bubble detection and not solid detection.

To conclude this part, it is not possible to firmly conclude that only the first high peak event in a group of peaks is a solid/tip interaction as for the highest solid loading and gas content no visualization are possible and solid/probe and bubble/probe interactions are a random process. However, as it will be shown later, from the available data, the comparison between the integrated void fraction and the bed expansion gives relatively accurate results and the error are in the range of the errors introduced by the probe itself even in two phase configurations. That is why we consider that, in the frame of this work, even if it is not perfect, the data processing strategy sounds consistent. In particular as the relative error is small between $\mathrm{TH}=1.1$ and normalized max amplitude (the range where events are either bubbles or solid/tip interactions) in the sequel, all the peaks will be considered when gas holdup profiles will be involved: this seems to be a reasonable approximation. Let us notice that concerning bubble velocity measurement, as the amplitude of the high intensity peaks is much higher than the regular amplitudes, the calibration curve, $V_{b}=f\left(T_{m}\right)$ is no longer valid as the rising time is computed between $10 \%$ and $90 \%$ of the mean "normal" gas level. So, in the calculation of the parameters where bubble rising velocity is involved all the high intensity peaks (normalized amplitude $>1.1$ ) must be rejected and velocities interpolated for these events.

\section{Results: application to three-phase flow measurements}

\subsection{Experimental set-up}

The measurements were performed in the same experimental set-up as in the previous section. No figure is presented for that experiment which is basically the same without vizualisation system for most experiments (except experiments dealing with bubbles sphericity). The on-line acquisition data was done using the software So2_4 developed at LEGI. As said in Section 2 these raw data are used to determine several gas phase characteristics, such as gas holdup and bubble velocity. The sampling rate was $100 \mathrm{KHz}$ and the duration of the acquisition is $300 \mathrm{~s}$. The optical probe was located at a height $h=28 \mathrm{~cm}$ from the gas sparger and measurements were performed varying the radial position. This radial positions were, respectively, $r=0, \pm 8, \pm 16, \pm 24, \pm 32 \mathrm{~mm}$. $r=0$ corresponds to the column axis.

The following solid loadings were used: $0,5,10,15,20,25$ and $30 \mathrm{vol} \%$. The clear (initial) liquid height was $H_{0}=0.295 \mathrm{~m}$ for all 
experiments (no liquid throughput). Experiments were done for the following four superficial gas velocities $1.6,2.7,3.8$ and $4.6 \mathrm{~cm} / \mathrm{s}$. The gas flow rate was red simultaneously from a rotameter and a mass flow meter. The global gas holdup was measured by measuring the water level height $H$ for each superficial gas velocities ("bed expansion" technique). It is assumed that values red from the flowmeter are precise. Concerning the global void fraction measurement, for homogeneous flow, the surface is stable and its height can be measured with a precision of $1 \mathrm{~mm}$ (resolution of the ruler used). As it will be seen later, the transition to heterogenous flow occurs for solid loading around $25 \%$ whatever the superficial gas velocity. In the range of (5-20\%) solids, the relative uncertainty on the void fraction measured by the liquid level expansion is around $0.6 \%$ for the highest void fraction and $3 \%$ for the lowest void fraction. When the transition has occurred, the surface start to wave and the uncertainty increase. The position of the interface is defined as a mean position

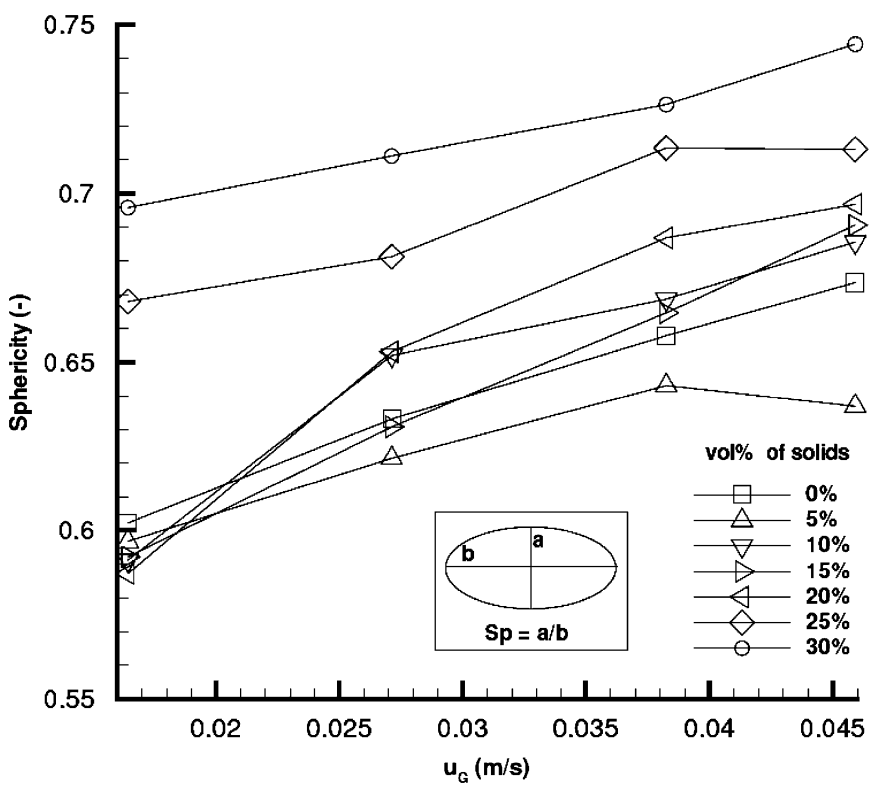

Fig. 14. Bubbles sphericity. over a sufficient number of period of the oscillations. Assuming that, with careful examination, this mean level can be determined with a precision of $3 \mathrm{~mm}$, the uncertainty is around 3\% for the highest gas holdup and increase up to $8 \%$ for the lowest gas holdup.

\section{2. preliminary results: visualization results-bubble sphericity}

As explained in Section 2.3, bubble interface/probe interactions are very important to quantify measurement errors. These interactions are closely related to bubble shape and interface oscillations or deformation. For the same conditions of the optical probe measurements presented below, images were recorded and treated in order to determine the bubble sphericity (Sp). Sp is defined in Fig. 14 as the ratio between the minor axis $a$ and the major axis $b$. The minimum and maximum bubble lengths were obtained from the picture analysis and the mean sphericity calculated from the ratio of these two quantities.

It can be seen in Fig. 14 that bubble sphericity increases with superficial gas velocity and solid loading. Furthermore, knowing the time residence $T_{G}$ and the bubble velocity, it is possible to access to the chord statistics (in particular the mean chord). An example of a mean chord profile is given in Fig. 15 for $u_{G}=2.7 \mathrm{~cm} / \mathrm{s}$. It is clear that the mean detected chord increases as soon as solids in added is the flow. The real size distribution itself is only accessible under strong assumption on the flow structure (Cartellier and Achard, 1991; Cartellier, 1999), and the transformation of the detected chord distribution into an actual diameter (or minor axe) size distribution is not an easy task even for narrow size distributions, spherical bubbles and homogeneous flow. Fig. 15 was then only shown as a clue about bubbles coalescence and the question of size distribution computation was beyond the scope of this paper. However, these results are consistent with previous works on the subject.

For instance, Mena et al. (2005b) showed that the introduction of a third phase promotes bubble interactions due to bubble-particle collisions. As the bubble coalescence rate increases, bigger and rounder bubbles are formed increasing the bubble sphericity. This change on bubble shape is not only important to interpret the behaviour of the profiles presented below. It will affect bubble interface/tip interaction which possibly can influence measurement accuracy.

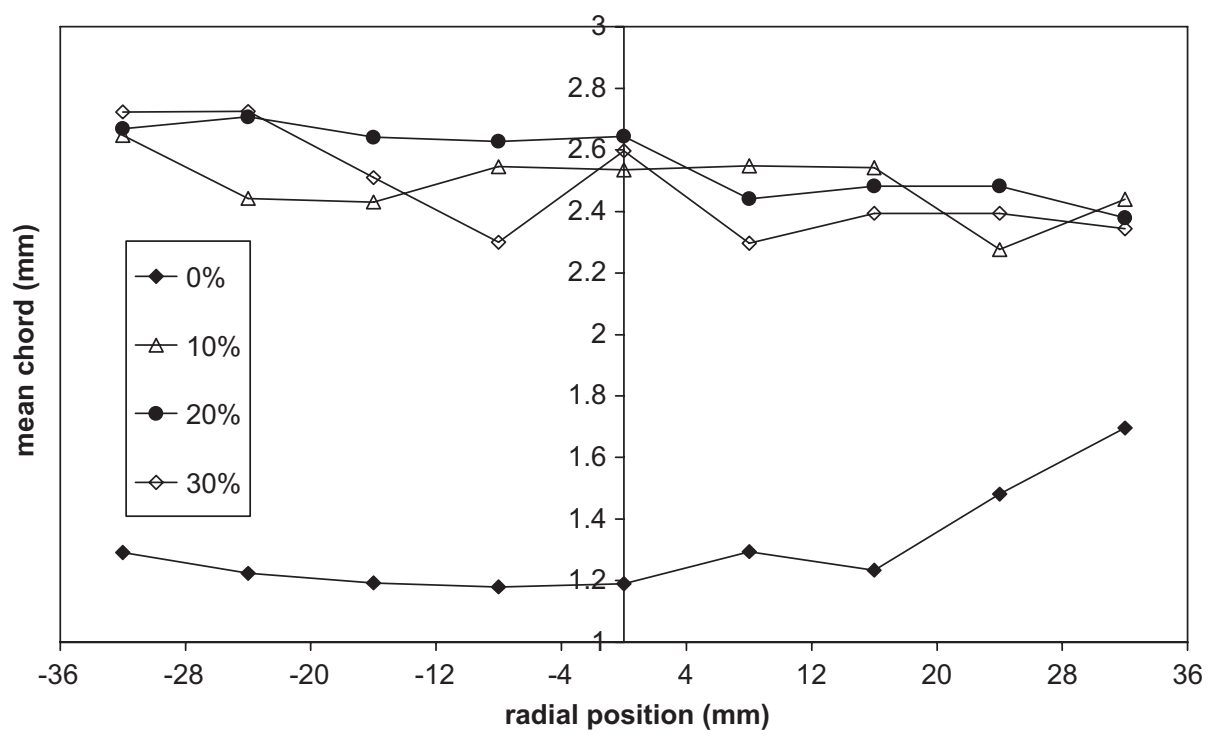

Fig. 15. Example of mean chord profiles for different solid loadings. $u_{G}=2.7 \mathrm{~cm} / \mathrm{s}$. 
a

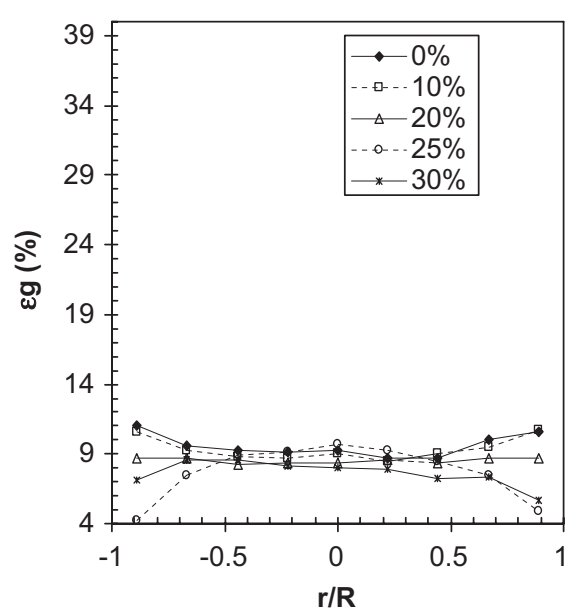

C

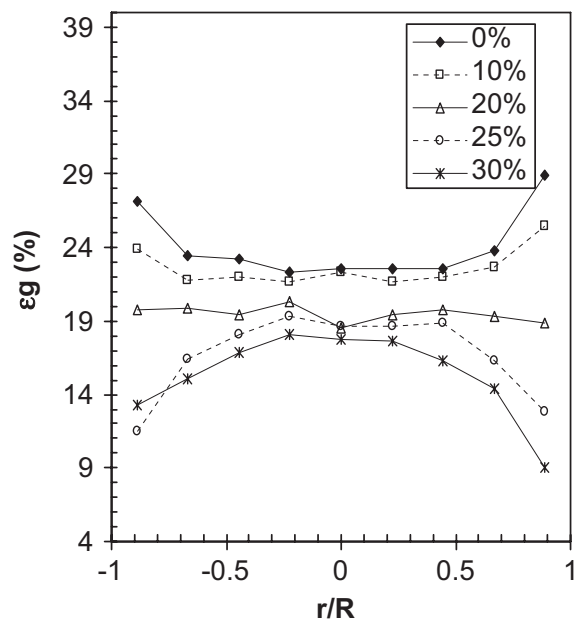

b

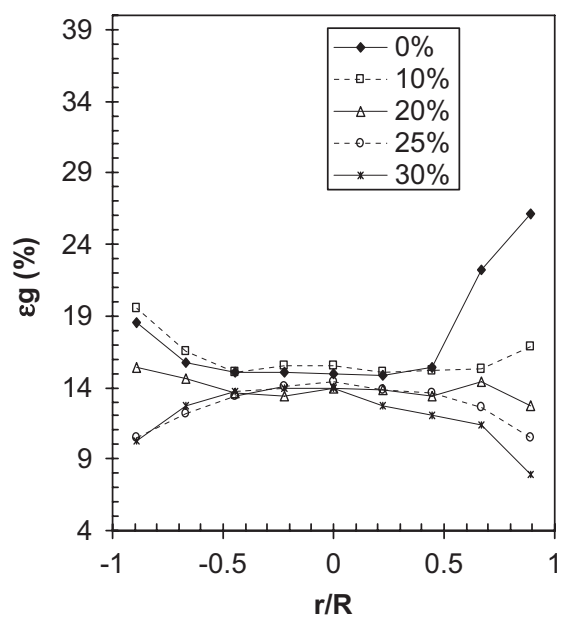

d

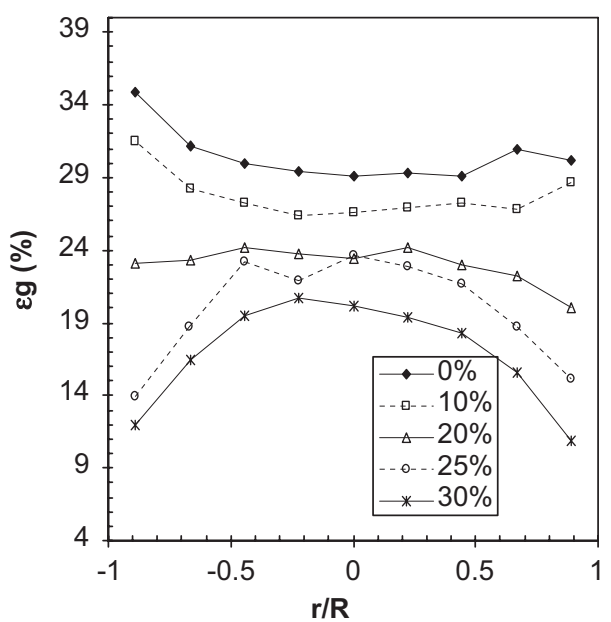

Fig. 16. Radial gas holdup profile for (a) $u_{G}=1.6 \mathrm{~cm} / \mathrm{s}$; (b) $u_{G}=2.7 \mathrm{~cm} / \mathrm{s}$; (c) $u_{G}=3.8 \mathrm{~cm} / \mathrm{s}$ and (d) $u_{G}=4.6 \mathrm{~cm} / \mathrm{s}$.

\subsection{Comparison between the optical probe results and global quantities}

Keeping in mind the bubble shape and size evolution with the solid loading, results obtained with the probe are presented and analysed.

\subsubsection{Void fraction profiles and mean gas holdup}

As mentioned before, one of the parameters obtained in the optical probe measurements is the residence time $T_{G}$, which represents the time the optical probe is in contact with the gas phase (when a bubble is pierced by the probe). The local gas holdup $(\varepsilon g)$ is defined as the ratio of the total residence time over the total acquisition time $\left(t_{\text {acq }}\right)$ (see Eq. (1)). The local gas holdup profiles were measured for the range of superficial gas velocities from 1.6 to $4.6 \mathrm{~cm} / \mathrm{s}$ and for solid loadings up to 30 vol\%. These profiles are presented in Fig. 16 and commented with physical arguments further in the text, Section 5.1.

In order to compare the probe results with other measurement techniques, these local gas holdup profiles were integrated over the column cross section to provide the mean gas holdup ( $\left.\varepsilon_{\text {mean }}\right)$ :

$\varepsilon_{\text {mean }}=\frac{1}{\pi R^{2}} \int_{0}^{R} \varepsilon_{g} 2 \pi r \mathrm{~d} r$.

The results of gas holdup obtained by optical probe and bed expansion are presented in Fig. 17. To determine $\varepsilon_{g}$ (optical probe), all the signal peaks have been considered (see Section 3.2). We also expect to be in the case of the poorest performance of the probe as our flow does not exhibit strong unidirectional flow (buoyancy driven flow).

For moderate solid loading (up to $20 \%$ ), the deviation between the two techniques seem rather low compared to the case with no solids, which could sound paradoxal. In fact, as the solid loading increase it has been shown previously that bubbles become bigger, rounder with a more stable shape. Their velocity increase leading to more favourable conditions for interface/tip interactions. There are more deviation at $0 \%$ loading as with no solids because, given the bubbles size, their shape and the oscillation of the interface must promote the various effects discussed in Section 2.3. Unfortunately, it is very difficult to quantify properly the contribution of each effects (blinding, drifting and crawling) on that experimental set-up. For the highest solid contents (25-30\%), the uncertainties increase. This is the case of the highest probability of solid-probe contact, but the deviation cannot be explained by false bubble detection as explained in Section 3.2. In these later configurations, stricto sensu, we are not anymore in a situation where the comparison between the integrated void fraction profiles and the volumetric void fraction is straightforward (3D flow with strong convection cells). However, the results were kept in Fig. 17 as a qualitative evaluation of the probe for these harsh operating conditions. Finally, the maximum and mean deviations between the results obtained with the two different techniques are, respectively, $22 \%$ and $7 \%$, but most of the measured 


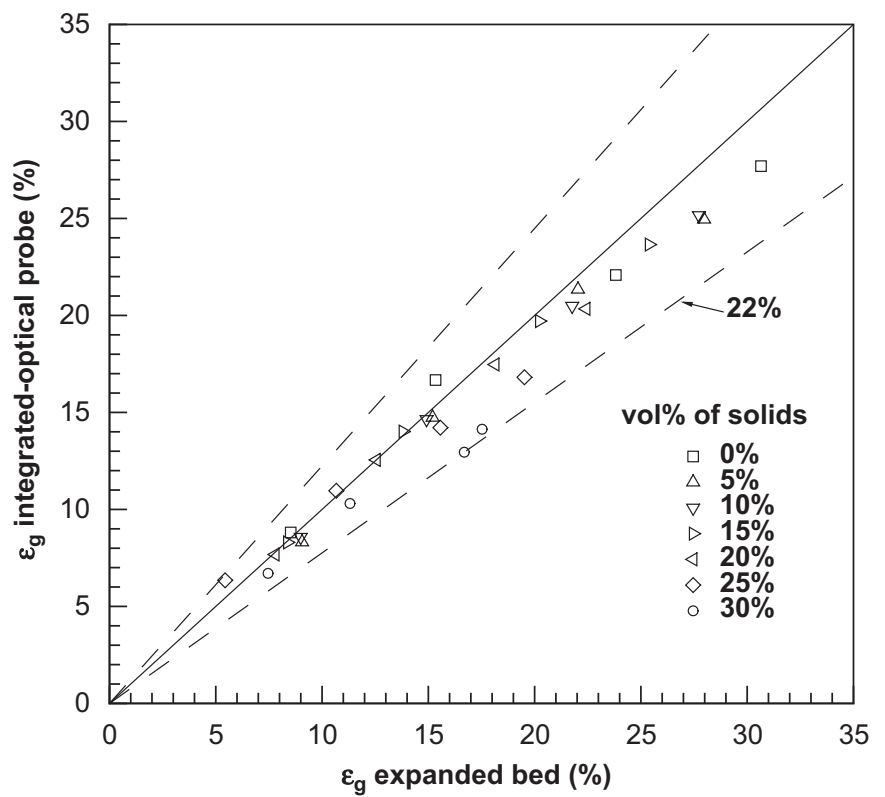

Fig. 17. Comparison between gas holdup obtained by optical probe and by bed expansion.

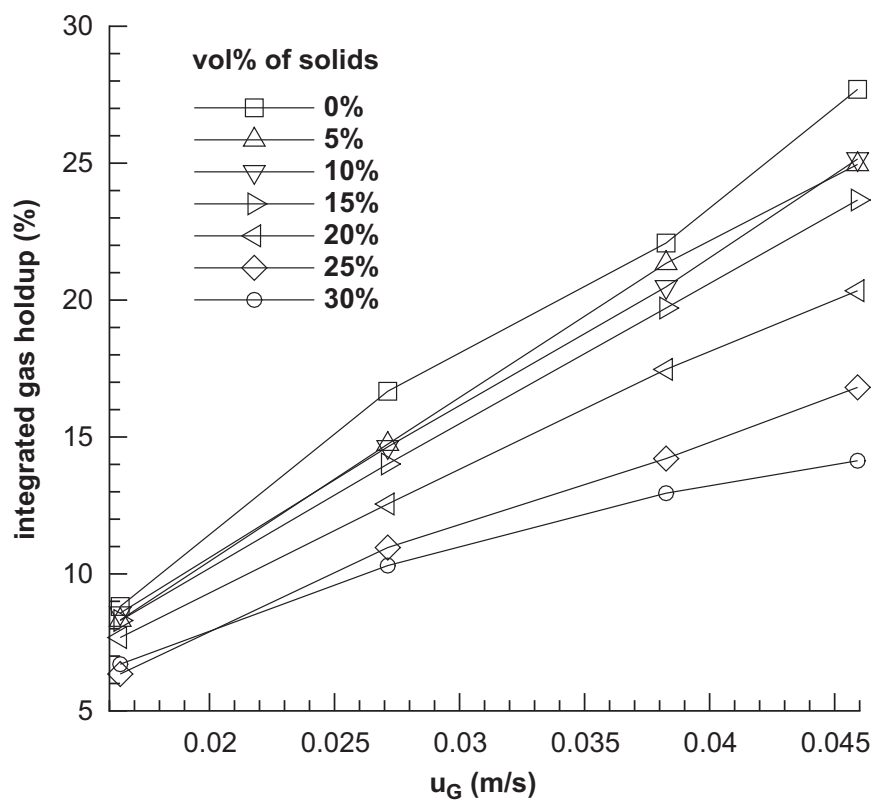

Fig. 18. Integrated gas holdup as a function of superficial gas velocity.

values gives errorslying in the range $\pm 10 \%$ which is neither better nor worst than errors commonly found with optical probes in twophase bubbly flow as well as flow which does not exhibit strong unidirectional flow and high absolute bubble velocities. Taking into account also the uncertainties due to the bed expansion technique, it is conclude that the probe can be used in three phase flow to measure relatively accurate void fraction profiles.

\subsubsection{Evolution of the gas holdup with the solid content}

Fig. 18 shows the evolution of the mean gas holdup with the superficial gas velocity and the solid loading, for the calcium alginate beads. The mean gas holdup increases almost linearly with the gas velocity, as expected. The increase of the solid content decreases the gas holdup and this effect is stronger for higher superficial gas
Table 1

Percentage of bubbles with measured $T_{m}: r=0$

\begin{tabular}{lllll}
\hline Percentage solids & $Q_{g}=4.4$ & $Q_{g}=6.6$ & $Q_{g}=9.3$ & $Q_{g}=11.2$ \\
\hline 0 & 84.6 & 85.16 & 82.82 & 80.09 \\
5 & 84.26 & 83.13 & 80.03 & 81.12 \\
10 & 83.73 & 82.62 & 80.58 & 81.31 \\
15 & 82.48 & 81.92 & 76.86 & 75.96 \\
20 & 81 & 78.27 & 69.69 & 68.016 \\
25 & 70.88 & 66.73 & 63.06 & 41.12 \\
30 & 68.76 & 65.17 & 59 & 50 \\
\hline
\end{tabular}

Table 2

Percentage of bubbles with measured $T_{m}: r=32 \mathrm{~mm}$

\begin{tabular}{lllll}
\hline Percentage solids & $Q_{g}=4.4$ & $Q_{g}=6.6$ & $Q_{g}=9.3$ & $Q_{g}=11.2$ \\
\hline 0 & 83.24 & 83.65 & 73.03 & 79.16 \\
5 & 84.08 & 85 & 78.99 & 72.29 \\
10 & 85.83 & 80.04 & 80.35 & 70.43 \\
15 & 83.09 & 78.72 & 75.93 & 70.15 \\
20 & 77.94 & 78.07 & 72.37 & 67.25 \\
25 & 71.45 & 72.35 & 66.74 & 62.98 \\
30 & 76.98 & 68.64 & 64.6 & 56.2 \\
\hline
\end{tabular}

velocities. This result is in agreement with previous works (Lu et al., 1995; Basini et al., 1995; De Swart et al., 1996; Reese et al., 1996; Thompson and Worden, 1997; Jianping and Shonglin, 1998; Gandhi et al., 1999; Luo et al., 1999; Fan et al., 1999). It is consistent also with a drag reduction as bubbles become rounder. Coupled with the phenomena of coalescence, despite bubble/solid interaction, bubble velocity thus increases and the overall void fraction decreases. This will be checked when gas velocity profiles will be analysed but these results give credit to the measurements performed by the probe.

\subsubsection{Velocity profiles and gas flow rate}

Bubble velocity is determined using a correlation between the rising time $T_{m}$ and the bubble velocity. This correlation is given by the probe calibration where the bubble velocity is estimated by a power function of $T_{m}\left(V=A * T_{m}^{B}\right)$. The calibration curve found for this particular optical probe is presented in Fig. 4.

This curve, measured in two-phase conditions, is intrinsic to the probe and can be used in 3 phases configuration as the calibration depends only on interface/tip interactions as seen in Section 2.3. However depending on the solid content and the way it affects the bubble shape as well as the interface deformations, solids can affect the error on the velocity measurement.

As stated before in Sections 2.2 and 3.2, (i) as the probe contamination comes along abnormal signal amplitudes the calibration curve is useless and the corresponding bubble velocities are interpolated for these events and (ii) for normal bubbles, only events whose signal follow a given criteria have their rising time calculated, the discarded events having their velocity interpolated. The last source of uncertainties comes from the validation rate of bubbles with measured rising time.

In Tables 1 and 2, the percentage of bubble with measured $T_{m}$ are shown for two positions in the column $(r=0$ and $32 \mathrm{~mm})$. Even with solid content, this percentage remains fairly high except for the highest solid loading (>25\%) and highest gas flow rate $(>9.3 \mathrm{Nl} / \mathrm{mn})$. This configurations corresponds to the case where in the column a complex 3D flow is encountered. So in most of the cases, the validation rate is sufficient to limit uncertainties on the mean velocity profiles.

Fig. 19 shows the bubble rise velocity profiles for the superficial gas velocities studied here.

Velocity profiles are in agreement with previous observations. As solid content increases, the profiles change progressively from flat to non-uniform. The introduction of a third phase promotes 
a

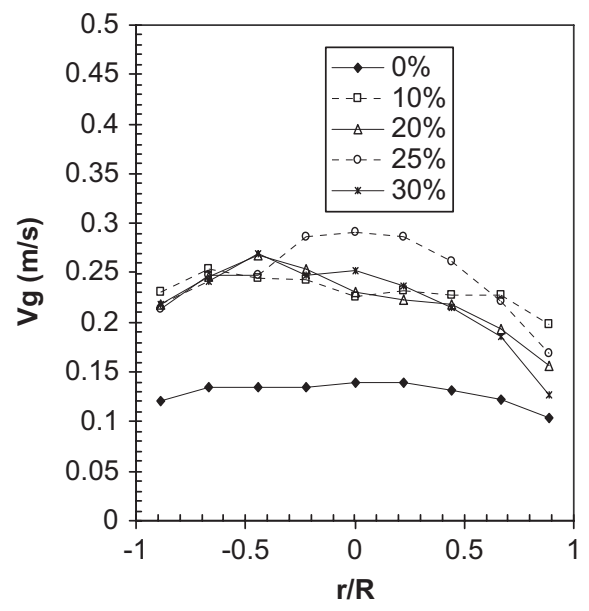

C

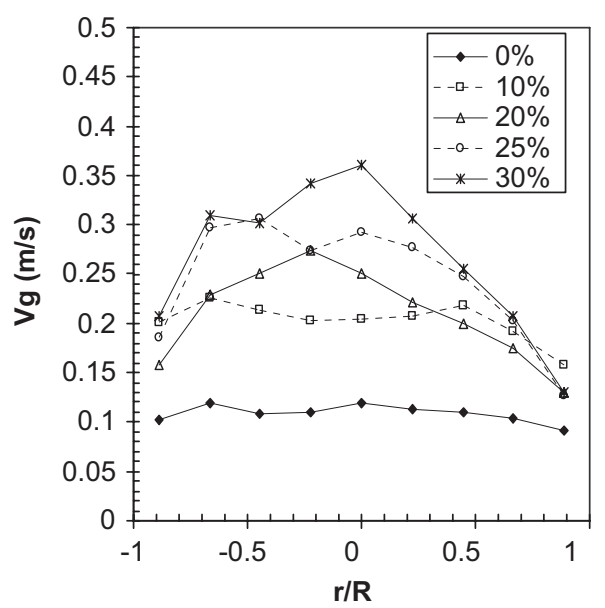

b

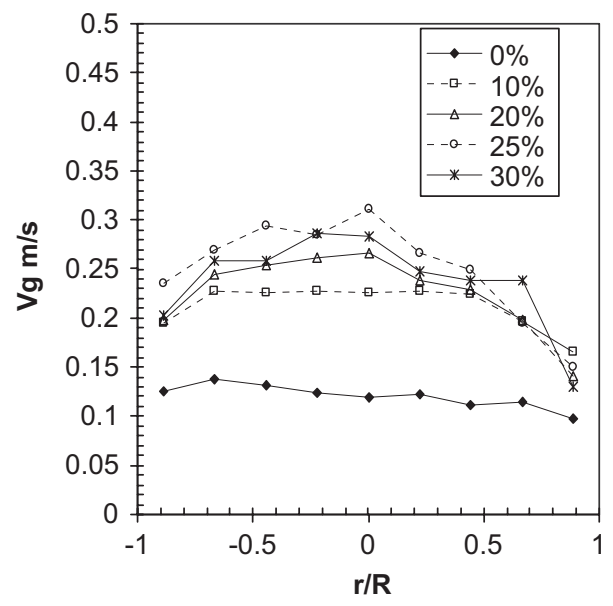

d

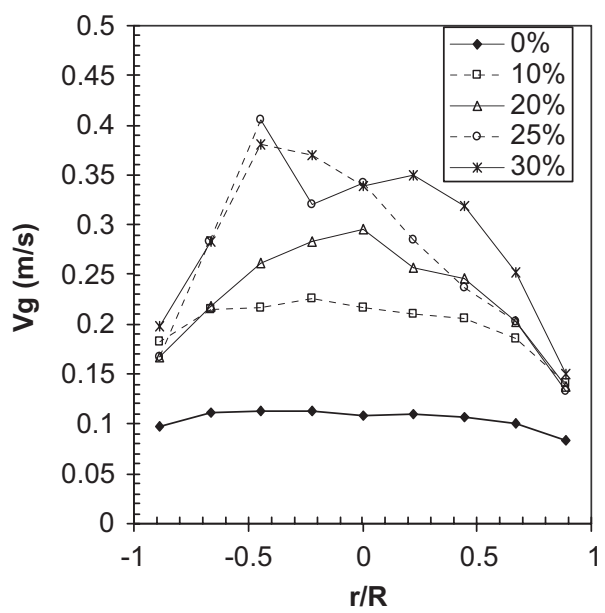

Fig. 19. Bubble rise velocity profile for (a) $u_{G}=1.6 \mathrm{~cm} / \mathrm{s}$; (b) $u_{G}=2.7 \mathrm{~cm} / \mathrm{s}$; (c) $u_{G}=3.8 \mathrm{~cm} / \mathrm{s}$ and (d) $u_{G}=4.6 \mathrm{~cm} / \mathrm{s}$.

bubble interactions due to bubble-particle collisions. Bubble coalescence rate is then increased resulting in bigger bubbles which rise faster and tendentiously through the centre of the column, yielding in a parabolic radial bubble velocity profile. This rise velocity increase comes probably along with a flow destabilization and the existence of strong recirculating cells: this would explain the parabolic shape of the profile. As no local liquid velocity measurements were performed, this last comment is at the present time only an assumption. The velocity increase due to coalescence and flow destabilization is particularly seen when profiles are compared with the $0 \%$ solid loading.

These observations are in agreement with Li and Prakash (2000) who also observed an increase of bubble rise velocity with solid loading and Wang et al. (2003) who denoted a slight change on the radial rise velocity profile as solid loadings increased.

Since the average gas holdup $(\varepsilon g)$ and bubble rise velocity $\left(V_{b}\right)$ are known along the column cross section, the bubble flow rate $\left(Q_{b}\right)$ can be computed as follows:

$Q_{b}=\int_{0}^{R} \varepsilon g v_{b} 2 \pi r \mathrm{~d} r$

In Fig. 20 the measured values of the bubble flow rate are compared with real gas flow rates (measured by a rotameter).

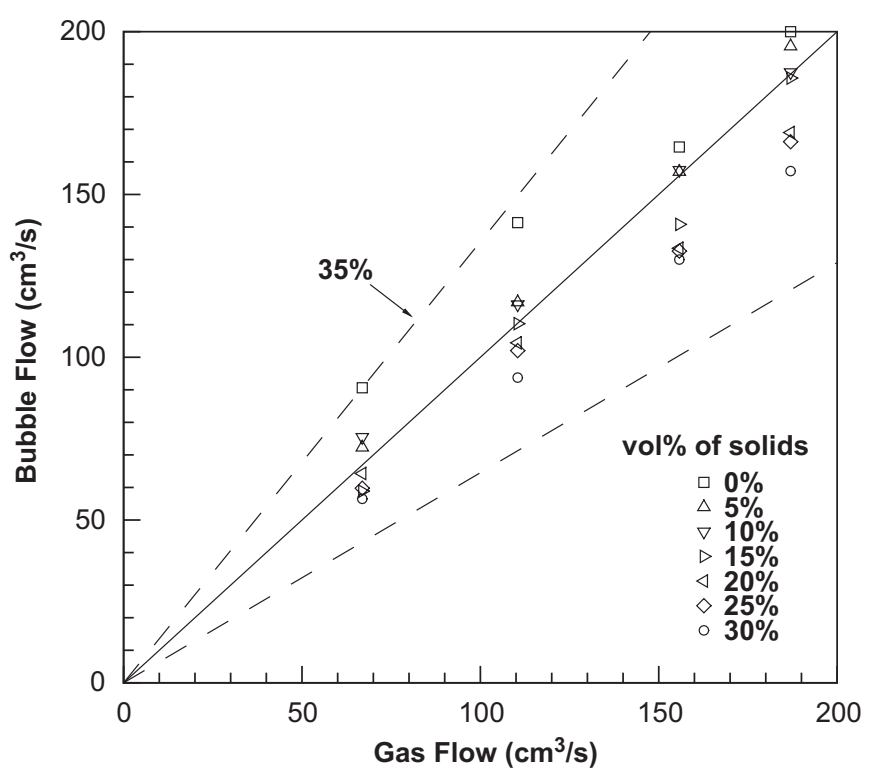

Fig. 20. Comparison between bubble flow and gas flow. 
Given the measurement error sources on the velocity measurements and residence time measurement, a rather fair agreement between bubble flow and gas flow was found, which suggests that the monofibre optical probe technique is suitable for gas holdup and bubble rise velocity local measurements. The maximum and mean deviations between bubble flow and gas flow is of $35 \%$ and $10 \%$, respectively. As seen for the gas holdup, deviations are maximum for no solid loading (and low gas superficial velocity). The physical explanation remains the same: higher solid loading gives bigger and rounder bubbles. At $0 \%$ and lower solid loading, bubble shape is more instable (flat bubbles, shape oscillation): for these conditions, even if the probe has a lower probability to pierce the interface on the "side" (which means greater impact angles), bubble piercing is not performed in ideal conditions leading to greater errors. Such effects are less pronounced at moderate solid loading as the bubble shape becomes more "stable". At higher solid contents and higher superficial velocity, as the structure of the flow can be complex with strong unsteady convection cells, the probe only detects bubbles on their upward motion and because of the complex bubble trajectories the error increases again.

Nevertheless, the results obtained on the average quantities with the optical probe are consistent with the results obtained with other techniques provided that the flow structure is not too complex which is the case for moderate solid loading and gas superficial velocity. In those case, the error lies in the same range as the values encountered for two-phase bubbly flows.

\subsubsection{Conclusion on the measurement of global variables}

Given expected error measurements from the probe, even in twophase flow, results obtained for three-phase flows remain in the range of encountered errors for other configurations. The agreement on global quantities deduced from the optical probe raw data and the same quantities measured by other means remains acceptable for the gas holdup and the gas flow rate.

Some global behaviours, for example the decrease of the mean gas holdup with the solid loading, are also in agreement with other experiments (Mena et al., 2005b). These experiments lend weight to the measurements conducted with the monofibre optical probe at least to recover global quantities describing the flow and their evolution with the operating conditions.

\section{Some perspectives on the potential use of monofibre optical probes in three-phase flow}

The results above are only preliminary results. The information available from the probes can give deeper information about the local flow structure. The experimental set-up used for this preliminary work was not designed for a detailed discussion on the local flow structure and would require a careful calibration. However, we present some results which are encouraging on the validation of the technique in three-phase flow systems.

\subsection{Transition between homogeneous and heterogeneous flow}

The local gas holdup profiles used in Section 4.2.1 for the mean gas holdup measurement are presented in Fig. 16. The figures show that the gas holdup profiles are strongly affected by the presence of solids. As the gas velocity increases, the influence of solid loading is even more relevant. For lower solid loadings (from 0 to $20 \mathrm{vol} \%$ ), the profiles are nearly flat and exhibit an increase near the wall. This increase can be attributed to the wall peaking effect (Rivière et al., 1999) and this effect is more pronounced for lower gas velocities. It seems that, for low solid loadings, the solid concentration increase attenuates the wall effect.
In the frame of this study, centred on the probe capacity, let us point out that it is mentioned in Deckwer (1992) that the wall effects are important for $D_{c} / d_{b}<20$. In our case, $D_{c} / d_{b}=0.072 / 0.005=14.4$ which confirms our previous expectations.

The physical explanation of the curve shape is difficult to fully understand without information on the liquid flow. For instance, bubble distributions in a two-phase flow depend on bubble-bubble interaction, deterministic force (as lift forces) as well as the induced agitation due to bubbles (Rivière et al., 1999). For three-phase configurations, radial bubble dispersion will depend on bubble/bubble and bubble/solid interactions and deterministic forces (as the lift force) will depend on the mean flow gradient as well as the bubble shape. Given the experimental results, for low to moderate solid loading, we assume that the wall peak attenuation is due to bubble dynamic modification (mainly bubble/bubble and solid/bubble interactions along bubble shape and size modification) as well as the increase of the liquid velocity fluctuations (induced by bubbles and solids). From $20 \%$ of solid content the profiles exhibit a negative parabolic curve with the maximum at the centre. As already explained, increasing further the solid loading (so increasing further bubble size) will ultimately leads to flow destabilization with parabolic void fraction profiles. This profile is consistent with the gas velocity profile shape. A better quantification of these various effects would require, however, simultaneous measurement of the flow field in the liquid. This was not the purpose of this paper.

The flat gas holdup radial profile is typical for the homogeneous flow regime (HoR) while the parabolic dependence is typical for the heterogeneous flow regime (HeR) (Ruzicka et al., 2001). This radial profile change clearly shows a transition of flow regimes due to the increase of the solid loading. For solid content between 0 and $20 \mathrm{vol} \%$ the homogeneous regime prevails while for higher solid concentrations the heterogeneous regime is dominant.

So, the local gas holdup profile, as obtained by the optical probe experiments, seems relevant in the identification of the flow regime and its transition. However, this technique requires a high experimental effort in a real application due to many radial distributed measurements.

\subsection{Specific gas-liquid interfacial area}

Another quantity of interest accessible through the probe is the specific gas-liquid interfacial area $(\Gamma)$. The determination of the specific gas-liquid interfacial area is essential to clearly understand the gas-liquid mass transfer behaviour of three-phase systems.

This quantity can be determined locally as

$\Gamma=4 \bar{f} \overline{\left(\frac{1}{V_{b}}\right)}$

where $\bar{f}$ is the mean bubble frequency which is defined as the ratio of the detected bubbles number $\left(n_{\text {det.bubbles }}\right)$ over the total acquisition time $\left(t_{\text {acq }}\right)$ :

$\bar{f}=\frac{n_{\text {det.bubbles }}}{t_{\text {acq }}}$

and $V_{b}$ is the bubble rise velocity. The specific gas-liquid interfacial area radial profiles were determined, but they show no monotonous dependence on the solid loading. The main limitation may be on the local bubble velocity measurement accuracy as the average of the inverse velocity of each individual event is involved in Eq. (4). This quantity do interfere more on the precision of the local specific interfacial area than the bubble frequency $\bar{f}$. Indeed, from the analysis presented in Section 3.2, it is shown that the local void fraction is fairly measured: so it is assumed that the total number of events is correctly evaluated. 


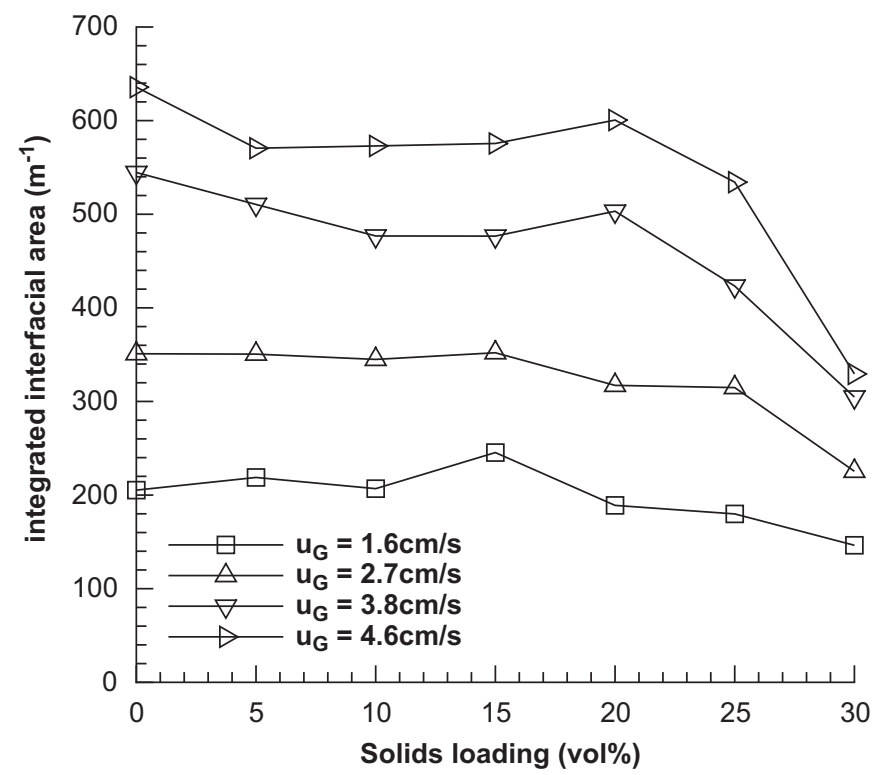

Fig. 21. Specific interfacial area as a function of the solid loading.

Even so, the local specific interfacial areas were integrated over the column cross section providing the mean specific interfacial area, $\Gamma_{\text {mean: }}$

$\Gamma_{\text {mean }}=\frac{1}{\pi R^{2}} \int_{0}^{R} \Gamma 2 \pi r \mathrm{dr}$.

The mean specific gas-liquid interfacial area is almost constant for solid concentrations up to $20 \mathrm{vol} \%$ and then decreases for further increases of the solid loading. This decrease is more pronounced for higher superficial gas velocities. The specific gas-liquid interfacial area was estimated in a previous work (Mena et al., 2005a) for the same three-phase system and for concentrations up to $10 \mathrm{vol} \%$. In that case, visualization experiments were performed followed by automatic image processing. It was observed that the specific interfacial area was not influenced by the solid particles, which agrees with the results of the present work. From this point, the optical probe seems also to be a promising tool to get local information on the three-phase flow.

To properly analyse physically the effect of solids on the interfacial area would require a refined study of the bubble size distribution evolution and bubbles velocities evolution with the solid loading. To perform that task, some theoretical problems must be overcome, in particular the bubbles size distribution reconstruction in three-phase flows conditions. This is beyond the scope of this paper. However, given the example of chords distributions presented in Fig. 15, the mean chord does not seem to change a lot with the solid loading and we can assume that the bubble size does not change "too much" either. The decrease on the interfacial area for the highest solid loading (around 20-25\%) would be then an effect of the fluid flow which would increase the absolute bubble velocity (leading to a decrease of the void fraction) for bubbles whose size is not too much different from one operating condition to another. This seems coherent with the fact that the heterogeneous regime (with 3D flows and strong convection cells) appears around this percentage of solid loading and it is consistent with the void fraction profiles already presented (Fig. 21).

\section{Conclusions}

For the first time in three-phase systems, local measurements of the gas phase characteristics were performed in a bubble column, using a monofibre optical probe. In spite of its fragile appearance, the probe proved to be very resistent even operating under high solid concentrations and gas flow rate conditions, therefore allowing to conduct long term measurements.

Frequently used in two-phase flows, the probe presented fair performance demonstrating that it could be a useful tool for the three-phase flow study. On the gas holdup, the maximum and mean deviations between the results obtained with two different techniques (optical probe and bed expansion) are $22 \%$ and $7 \%$, respectively, for that quantity. The maximum deviation occurs for the heterogenous regime where the comparison between the integrated void fraction profile and gas holdup measured by bed expansion is not straightforward.

Taking into account the error introduced by the probe on the velocity measurement, the integration of the velocity profiles gave a mean deviation around $10 \%$ which corresponds to typical uncertainties even in two-phase flows configuration for that quantity. The maximum deviation on the gas flux (35\%) occurs for no solid loading. This phenomena is explained by the change in the bubble shape and interface stability: with no solids, given the bubbles size, the interface is subject to oscillation and deformation. With solids, bubbles becomes rounder with a more stable shape: as the velocity measurement accuracy is mainly controlled by the impact angle between the probe tip and the interface, these later conditions are more favourable.

The experimental results show that the radial gas holdup profile shape is affected by the presence of the solid phase and the mean gas holdup decreases as the solid content increases. It was observed that up to $20 \mathrm{vol} \%$ of solid concentration a flat gas holdup profile occurs, with an increase near the wall. For solid concentrations higher than 20 vol\%, a negative parabolic gas holdup profile was observed suggesting an homogeneous-heterogeneous flow regime transition. The overall gas holdup decrease with the solid content sounds physically consistent with bubbles coalescence induced by the solid phase. This assumption is confirmed by vizualizations which show an increase of bubbles sphericity with the solid loading and is also confirmed by chord measurements which exhibit increase of the mean chord value as soon as solid is introduced in the flow.

Bubble rise velocity radial profiles change from flat to nonuniform profiles when the solid content is increased. These observations are also characteristic of a regime transition. In the same way, the mean gas-liquid interfacial area evolution with the solid loading exhibits the same tendency than other results obtained in other experiments by image processing: the solid loading does not affect the mean interfacial area except for heterogenous flows. In that case, the strong convection cells increase the absolute bubble velocity, decrease the gas holdup and results in a decrease of the interfacial area.

All these results (transition, bubble coalescence) are consistent with results from other experiments obtained by alternative measurement techniques. Thus, the probe proved its capacity to measure some characteristics of the three-phase flows.

However, limitations remain. On the global variable, such as the gas holdup, the precision is fair compared to other techniques but the measurements require a high experimental effort. This could be not sustainable in real applications. Supplemented with other measurement techniques, this probe could be useful for fundamental studies on laboratory scales pilot and a help to understand such complex flows where coupling between phases is strong. The problem of solid/probe interactions and their effects on the signal remains an open question even if some answers were given in that paper for alginate beads. Finally, as global quantities are dependant on the solid/bubble/probe tip interactions (blinding effect for instance for the void fraction measurement, bubble deformation and interface piercing for the velocity), more systematical 
studies should be undertaken on these points to fully evaluate the technique.

Finally, one of the interests of the probe consists in measuring at the same time the residence time and velocity of each individual events, allowing to access the chord distribution. The chord distribution example presented in this paper showed no strong evolution with the solid loading. Apart from the problem of the real size distribution reconstruction from the detected chord distribution, the accuracy on the velocity and residence time of individual events have to be improved in this complex flows in order to fully exploit the probe capacity.

All these studies were beyond the scope of this paper, aiming at checking the potential use of monofibre optical probes in three-phase flow systems. The preliminary results presented show that provided some limitations better understood, it could be an interesting tool to study the behaviour of three phase reactors, for example those used in bioengineering, such as airlift with immobilized biomass.

\section{Acknowledgements}

This work was supported by Fundacção para a Ciência e Tecnologia under program Contract number SFRH/BD/3427/2000 and Contract POCTI/EQU/45194/2002. The authors would like to thank Laurent Terrier for his precious technical assistance.

\section{References}

Barrau, E., Riviere, N., Poupot, C., Cartellier, A., 1999. Single and double optical probes in air-water two-phase flows: real time signal processing and senso performance. International Journal of Multiphase Flow 25

Basini, S., Finch, J.A., Laplante, A.R., Weber, M.E., 1995. Effect of solid particles on gas holdup in flotation column-i. Measurement. Chemical Engineering Science 50, 2329-2334.

Boyer, C., Cartellier, A., 1999. Bubble velocity and size estimation using a single optica probe in a gas/liquid flow across a fixed bed reactor. ECCE, vol. 2, Montpellier

Boyer, C., Duquenne, A.-M., Wild, G., 2002. Measuring techniques in gas-liquid and gas-liquid-solid reactors. Chemical Engineering Science 57, 3185-3215.

Carrica, P., Delgadino, D., Marco, Z.D.D., 1995. In: Celata, G., Shah, R. (Eds.), TwoPhase Flow Modelling and Experimentation. Edizioni ETS

Cartellier, A., 1992. Simultaneous void fraction measurement, bubble velocity, and size estimate using a single optical probe in gas-liquid two-phase flow. Review of Scientific Instruments 63, 5442-5453.

Cartellier, A., 1998. Measurement of gas phase characteristics using new monofibre optical probes and real time signal processing. Nuclear Engineering and Design 184, 393-408.

Cartellier, A., 1999. Post-treatment for phase detection probes in non uniform twophase flows. International Journal of Multiphase Flow 25, 201-228.

Cartellier, A., Achard, J., 1991. Local phase detection probes in fluid/fluid flow. Review of Scientific Instruments 62, 279-303.

Cartellier, A., Barrau, E., 1998a. Monofibre optical probes for gas detection and gas velocity measurements: conical probes. International Journal of Multiphase Flow $24,1265-1294$

Cartellier, A., Barrau, E., 1998b. Monofibre optical probes for gas detection and gas velocity measurements: optimised sensitive tip. International Journal of Multiphase Flow 24, 1295-1315.

Cartellier, A., Rivière, N., 2001. Bubble-induced agitation and microstructure in uniform bubbly flows at small to moderate particle Reynolds numbers. Physics of Fluids 13, 2165-2181.

De Lasa, H., Lee, S.L.P., Bergougnou, M.A., 1984. Bubble measurement in threephase fluidized beds using a u-shape optical fibre. Canadian Journal of Chemica Engineering 62, 165-169.

De Swart, J.W.A., Van Vliet, R.E., Krishna, R., 1996. Size, structure and dynamics of "large" bubbles in a two-dimensional slurry bubble column. Chemical Engineering Science 51, 4619-4629.

Deckwer, W.D., 1992. Bubble Column Reactors. Wiley, Chichester.

Fan, L.-S., Yang, G.Q., Lee, D.J., Tsuchiya, K., Luo, X., 1999. Some aspects of highpressure phenomena of bubbles in liquids and liquid-solid suspensions. Chemical Engineering Science 54, 4681-4709.

Fordham, E.J., Holmes, A., Ramos, R.T., Simonian, S., Huang, S.-M., Lenn, C.P., 1999 Multiphase fluid discrimination with local fibre optical probes: I liquid-liquid flow. Measurement Science \& Technology 10, 1329-1337.

Freitas, C., Teixeira, J.A., 2001. Oxygen mass transfer in a high solids loading threephase internal-loop airlift reactor. Chemical Engineering Journal 84 (1), 57-61.
Gandhi, B., Prakash, A., Bergougnou, M., 1999. Hydrodynamic behaviour of slurry bubble column at high solids concentrations. Powder Technology 103 80-94.

Hong, M., Cartellier, A., Hopfinger, E., 2004. Characterization of phase detection optical probe for the measurement of the dispersed phase parameters in sprays. International Journal of Multiphase Flow 30, 615-648.

Jianping, W., Shonglin, X., 1998. Local hydrodynamics in a gas-liquid-solid threephase bubble column reactor. Chemical Engineering Journal 70, 81-84.

Joly-Vuillemin, C., de Bellefon, C., Delmas, H., 1996. Solid effects on gas-liquid mass transfer in three-phase slurry catalytic hydrogenation of adiponitrile over Raney nickel. Chemical Engineering Science 51, 2149-2158.

Julia, J.E., Harteveld, W., Mudde, R.F., den Akker, H.V., 2005. On the accuracy of void fraction measurements using optical probes in bubbly flows. Review of Scientific Instruments 76

Kiambi, S.L., Duquenne, A.M., Bascoul, A., Delmas, H., 2001. Measurements of local interfacial area: application of bi-optical fibre technique. Chemical Engineering Science 56, 6447-6453.

Kozma, R., 1995. Studies of the relationship between the statistics of void fraction fluctuations and the parameters of two-phase flows. International Journal of Multiphase Flow 21, 241-251.

Leung, W., Revankar, S., Ishii, Y., Ishii, M., 1995. Axial development of interfacial area and void concentration profiles measured by double-sensor probe method. International Journal of Heat and Mass Transfer 38 (3), 445-453.

Li, H., Prakash, A., 2000. Influence of slurry concentrations on bubble population and their rise velocities in a three-phase slurry bubble column. Power Technology $113,158-167$

Liu, W., Clark, N.N., Karamavruç, A.I., 1998. Relationship between bubble size distributions and chord-length distribution in heterogeneously bubbling systems. Chemical Engineering Science 53 (6), 1267-1276.

Lu, W., Hwang, S., Chang, C., 1995. Liquid velocity and gas holdup in three-phase internal loop airlift reactors with low-density particles. Chemical Engineering Science 50, 1301-1310.

Luo, X., Lee, D.J., Lau, R., Yang, G., Fan, L.-S., 1999. Maximum stable bubble size and gas holdup in high-pressure slurry bubble columns. A.I.Ch.E. Journal 45, 665-680.

Mena, P.C., Pons, M.N., Teixeira, J.A., Rocha, F.A., 2005a. Using image analysis in the study of multiphase gas absorption. Chemical Engineering Science 60, $5146-5152$.

Mena, P.C., Ruzicka, M.C., Rocha, F.A., Teixeira, J.A., Drahos, J., 2005b. Effect of solids on homogeneous-heterogeneous flow regime transition in bubble columns. Chemical Engineering Science 60, 6013-6026.

Reese, J., Jiang, P., Fan, L., 1996. Bubble characteristics in three-phase systems used for pulp and paper processing. Chemical Engineering Science 51, 2501-2510.

Rivière, N., Cartellier, A., Timkin, L., Kashinsky, O., 1999. Wall shear stress and void fraction in poiseuille bubbly flows: part i and ii. European Journal of Mechanics B-Fluids 18, 823-867.

Ruzicka, M.C., Zahradnik, J., Drahos, J., Thomas, N.H., 2001. Homogeneousheterogeneous regime transition in bubble columns. Chemical Engineering Science 56, 4609-4626.

Sada, E., Kumazawa, H., Lee, C., Fujiwara, N., 1985. Gas-liquid mass transfer characteristics in a bubble column with suspended sparingly soluble fine particles. Industrial \& Engineering Chemistry Process Design and Development 24, 255-261.

Schweitzer, J.-M., Bayle, J., Gauthier, T., 2001. Local gas hold-up measurements in fluidized bed and slurry bubble column. Chemical Engineering Science 56, $1103-1110$

Silva, M.L.D., Schleicher, E., Hampel, U., 2007. A novel needle probe based on high speed complex permittivity measurements for investigation of dynamic fluid flows. IEEE Transaction on Instrumentation and Measurements 56 (4), 1249-1256.

Thompson, V.S., Worden, R.M., 1997. Phase holdup, liquid dispersion, and gasto-liquid mass transfer measurements in a three-phase magnetofluidized bed. Chemical Engineering Science 52, 249-295

Wang, T., Wang, J., Yang, W., Jin, Y., 2003. Experimental study on bubble behaviour in gas-liquid-solid three-phase circulating fluidized beds. Powder Technology 137, 83-90.

Warsito, W., Ohkawa, M., Kawata, N., Uchida, S., 1999. Cross-sectional distributions of gas and solid holdups in slurry bubble column investigated by ultrasonic computed tomography. Chemical Engineering Science 54, 4711-4728.

Warsito, W., Fan, L.S., 2001. Measurement of real-time flow structures in gas-liquid and gas-liquid-solid flow systems using electrical capacitance tomography (ECT). Chemical Engineering Science 56, 6455-6462.

Xie, T., Ghiaasiaan, S., Karrila, S., McDonough, T., 2003. Flow regimes and gas holdup in paper pulp-water-gas three-phase slurry flow. Chemical Engineering Science $58,1417-1430$.

Yang, W., Wang, J., Wang, T., Jin, Y., 2001. Experimental study on gas-liquid interfacial area and mass transfer coefficient in three-phase circulating fluidized beds. Chemical Engineering Journal 84, 485-490.

Zhang, J.P., Grace, J.R., Epstein, N., Lim, K.S., 1997. Flow regime identification in gas-liquid flow and three-phase fluidized beds. Chemical Engineering Science 52, 3979-3992. 